The AstrophysiCAL Journal, 460:252-270, 1996 March 20

(C) 1996. The American Astronomical Society. All rights reserved. Printed in U.S.A.

\title{
NUCLEAR RINGS IN ACTIVE GALAXIES
}

\author{
ThAISA STORCHI-BERGMANN ${ }^{1}$ \\ Departamento de Astronomia, Instituto de Fisica, Universidade Federal de Rio Grande de Sul, CP 15051, CEP 91501-970, Porto Alegre, RS, Brazil \\ ANDREW S. WILSON ${ }^{1}$ \\ Astronomy Department, University of Maryland; College Park, MD 20742; and Space Telescope Science Institute \\ AND \\ JACK A. BALDWIN \\ Cerro Tololo Inter-American Observatory, National Optical Astronomy Observatories, Casila 603, La Serena, Chile \\ Received 1995 May 5; accepted 1995 August 17
}

\begin{abstract}
We investigate two current problems in active galactic nuclei- the mode of fueling the putative black hole, and the question of whether the circumnuclear regions have experienced unusual chemical processing - by studying the kinematics and chemical abundance of the gas in the inner regions of active galaxies with circumnuclear star formation. We discuss results for three galaxies with nuclear rings: NGC 1097 and NGC 1672, both of which contain a LINER nucleus, and NGC 5248, a galaxy with a ring but no nuclear activity. Narrowband images have been obtained in $\mathrm{H} \alpha+[\mathrm{N} \mathrm{II}]$, to map out the ionized gas. Medium-dispersion long-slit spectroscopy at several positions over the nuclear region has been used to obtain the gaseous velocity field and the distribution of excitation. The gaseous kinematics indicate large central mass concentrations in the three galaxies. The two galaxies with LINER nuclei appear to have two inner Lindblad resonances, with the star-forming rings located between them. The high sensitivity of the data has allowed us to detect faint ionized gas with high $[\mathrm{N} \mathrm{II}] / \mathrm{H} \alpha$ ratios-a warm ionized medium - up to several kiloparsecs from the nuclei. We find a correlation between the $[\mathrm{N} \mathrm{II}] / \mathrm{H} \alpha$ and $[\mathrm{S} \mathrm{II}] / \mathrm{H} \alpha$ ratios and the $\mathrm{FWHM}$ of the $[\mathrm{N} \mathrm{II}] \lambda 6584$ emission line. This correlation is strongest for the circumnuclear gas but is also present in the warm ionized medium of the two galaxies with LINER nuclei, suggesting that shocks contribute to the ionization of the gas. From long-slit spectroscopy at low dispersion, we have obtained the chemical composition of the $\mathrm{H}$ II regions in the ring and far beyond. The highest central metallicities $(\mathrm{O} / \mathrm{H} \approx 2$ times solar and $\mathrm{N} / \mathrm{O} \approx 3$ times solar) were found around the two LINER nuclei, confirming previous results based on spectroscopy of the narrow-line region that LINER nuclei have abundances considerably in excess of solar. Nevertheless, these abundances are similar to those of the nuclei of non-active galaxies with the same morphological type and absolute magnitude.
\end{abstract}

Subject headings: galaxies: abundances — galaxies: active — galaxies: ISM galaxies: kinematics and dynamics — galaxies: nuclei — galaxies: structure

\section{INTRODUCTION}

The canonical model for an active galactic nucleus (AGN) comprises a supermassive black hole accreting material from its surroundings. Arguments based on total radiated luminosity over the lifetime of the AGN indicate that the amounts of material ("fuel") which must be accreted are so large as to require a galaxy wide supply. The problem is then to remove essentially all the angular momentum of the gas so that it can fall the eight or more orders of magnitude in radius from the galaxy scale to a few hundred gravitational radii of the black hole, where kinematic viscosity is believed to take over the outward transport of angular momentum. The most promising suggestions for processes which allow gas to fall in from galaxy-wide to circumnuclear $(\approx 100 \mathrm{pc})$ scales are oval distortions or bars in the gravitational potential of the galaxy and galaxy-galaxy interactions. Such processes are expected to induce morphological and kinematical signatures in both the stars and gas, and these signatures can be useful probes

\footnotetext{
${ }^{1}$ Visiting Astronomer at Cerro Tololo Inter-American Observatory, operated by the Association of Universities for Research in Astronomy, Inc., under contract with the National Science Foundation.
}

of the fueling mechanism, especially if they are observed close to the active nucleus.

Recent programs of imaging and spectroscopy have revealed circumnuclear $(<1 \mathrm{kpc})$ rings in a number of Seyfert galaxies (e.g., Pogge 1989; Wilson et al. 1991; Genzel et al. 1995). These rings are sites of active star formation, as evidenced by strong, low-excitation emission lines and blue continuum colors. In the few cases for which the rotation curve of the galaxy has been measured, the rings are located very close to the turnover point in the rotation curve (Wilson et al. 1986; Telesco \& Decher 1988). For a conventionally shaped rotation curve (inner solid body plus outer flat part), the quantity $\Omega-\kappa / 2$ is a maximum at the turnover and then falls off further out, allowing the possible existence of an inner Lindblad resonance (ILR), where $\Omega_{p}=\Omega-\kappa / 2$ (e.g., Kormendy \& Norman 1979). Here the usual notation is used: $\Omega$ represents the angular frequency of the gas and stars; $\kappa$, the epicyclic frequency; and $\Omega_{p}$ the pattern speed. It is then plausible to associate rings near turnover with an ILR. Indeed, this is the "conventional" explanation for nuclear rings in early to intermediate barred galaxies (see, e.g., Buta 1987); models (see, e.g., Schwarz 1981; Piner, Stone, \& Teuben 1995) reveal how dynamical 
resonances between orbital motions and the pattern speed of a bar or oval distortion lead to ringlike concentrations of gas which give stellar rings after star formation. The models of Simkin, Su, \& Schwarz (1980) show strong gaseous inflow in association with an ILR. These authors argued that this inward transport may be self-sustaining since the continuous pile-up of gas increases the central density and maintains the ILR.

In order to investigate further the kinematics of nuclear rings in active galaxies, we have obtained mediumdispersion long-slit spectroscopy covering the spectral region around $H \alpha$ for a sample of active galaxies with starforming nuclear rings. Our goal is to measure the gaseous velocity field and thus check whether the rings may be associated with an ILR, and also to study in detail the kinematics of the rings themselves.

A related area of interest concerns the relationship between star formation and nuclear activity. Ideas cover a wide range, from models in which no black hole is present and the entire Seyfert phenomenon is a starburst in a metalrich environment (e.g., Terlevich 1990), to those in which the starburst and black hole activity seem related only because of a common fueling mechanism. If Seyfert galaxies have suffered unusually large amounts of massive star formation over their lifetimes in their central regions, enhancement of heavy element abundances in comparison with "normal" spirals might be expected. Indeed, previous studies of the emission-line ratios of $\sim 180$ Seyfert 2 and LINER nuclei have led to the conclusion that an enhanced nitrogen abundance, from near solar to $\sim 5$ times solar, is present (Storchi-Bergmann \& Pastoriza 1989, 1990). However, abundance determinations from the emission lines of the narrow-line region are subject to various uncertain physical processes, such as heating by shocks, which can increase the [N II] $6584 / \mathrm{H} \alpha$ ratio. It is much more secure to use $\mathrm{H}$ II regions close to the nuclei, for which standard methods (e.g., McCall, Rybski, \& Shields 1985; Dopita \& Evans 1986) of abundance determination can be followed. We have, therefore, also observed the circumnuclear regions (especialy the rings) of the sample galaxies with low-dispersion long-slit spectroscopy in order to obtain key line flux ratios over the entire optical spectrum and calculate chemical abundances. Through the long-slit spectroscopy, we were also able to observe a number of $\mathrm{H}$ II regions in the disks of the galaxies, allowing the derivation of radial abundance gradients.

Finally, the excellent sensitivity of our mediumdispersion spectra has allowed the detection of emission lines, not only from the nuclei, rings, and $\mathrm{H}$ II regions, but also from diffuse gas apparently in the disks of the galaxies. Similar emission has been observed in the Milky Way-the Reynolds layer - and in a few external galaxies, and the diffuse gas producing it has been called the warm ionized medium (hereafter WIM) (e.g., Rand 1994 and references therein). We discuss the excitation of the WIM on the basis of the observed emission-line ratios and widths.

In the present paper, we present the results on the kinematics, gaseous excitation, and chemical abundances of three galaxies with nuclear rings: NGC 1097, NGC 1672, and NGC 5248.

\section{THE GALAXIES OBSERVED}

The three galaxies were selected as having nuclear rings, similar morphological types ( $\mathrm{Sb}$ or $\mathrm{Sbc}$ ) and a suitably inclined orientation on the sky for kinematical study. NGC
1097 and NGC 1672 are barred, and NGC 5248 is classified as SAB. They are members of the sample of "peculiar nuclei" of Sérsic \& Pastoriza (1965): the nuclei of NGC 1097 and NGC 5248 are classified as " hot spot" and that of NGC 1672 as "amorphous." NGC 1097 and NGC 5248 are also included in the list of galaxies with nuclear rings recently compiled by Buta \& Crocker (1993) on the basis of continuum imaging. The absence of NGC 1672 from this list may probably be ascribed to the fact that its ring can be clearly observed only in line emission (see $\$ 4.1$ ) and is inconspicuous in continuum light.

The nuclear ring (or tight spiral) in NGC 1097 (Hummel, van der Hulst, \& Keel 1987; Forbes et al. 1992) surrounds a LINER nucleus (Phillips et al. 1984), in which very broad, double-peaked Balmer emission has recently appeared (Storchi-Bergmann, Baldwin, \& Wilson 1993). The broad nuclear lines in this galaxy are probably related to the remarkable large-scale optical jets in this galaxy (Wolstencroft \& Zealey 1975; Arp 1976; Lorre 1978) that extend to $90 \mathrm{kpc}$ from the nucleus.

The nuclear ring in NGC 1672 also surrounds a nucleus with low-level activity (Véron-Cetty \& Véron 1986), while NGC 5248, which does not harbor an active nucleus was selected for a comparison.

We have collected (mostly from the literature) in Table 1 relevant information on these three galaxies: morphological types, distances, absolute magnitudes, inclinations, position angles of the major axes and of their bars, scale (parsecs per arcsecond) at the galaxy, and the major and minor axis diameters of the nuclear rings. Distance-dependent quantities have been calculated assuming a Hubble constant of $H_{0}=75 \mathrm{~km} \mathrm{~s}^{-1} \mathrm{Mpc}^{-1}$. The references for these data are listed at the end of the table.

\section{OBSERVATIONS AND REDUCTIONS}

Direct images of the three galaxies were obtained at the $1.5 \mathrm{~m}$ telescope of the Cerro Tololo Inter-American Observatory (CTIO) in 1991 October and 1992 May, using a CCD detector and a set of narrowband filters centered on the $\mathrm{H} \alpha$ line (plus [N II] $\lambda \lambda 6548,6584$ “on-band") and adjacent continua ("off-band"). A $\log$ of the observations is shown in Table 2. The images were reduced using standard procedures in IRAF. The images in the on- and off-band filters were aligned using field stars. The on- and off-band images of NGC 5248 have different spatial scales, so the field stars were used to interpolate the on-band image to the scale and orientation of the off-band image. Each off-band image was then scaled by a factor such that the integrated number of counts for the stars in the field was equal to the corresponding value in the adjacent on-band image. Finally, images in $\mathrm{H} \alpha+[\mathrm{N}$ II $]$ were produced by subtracting the off- from the on-band image.

Long-slit spectroscopic observations were obtained using a CCD detector on the Ritchey-Chrétien Spectrograph at the CTIO $4 \mathrm{~m}$ telescope, through a $2^{\prime \prime}$ slit. A log of these observations is given in Table 3 . Two different setups were used: medium-dispersion (1.7 $\AA$ resolution FWHM) spectroscopy in the red to study the kinematics, and lowdispersion ( $8 \AA$ resolution) spectroscopy covering most of the optical range to measure the physical properties and chemical abundance of the gas. The orientations of the slit were selected from the images to optimize the coverage of the nuclear rings and $H$ II regions at larger radii. Thus, the slit was not, in general, aligned with the parallactic angle. 
TABLE 1

BASIC DATA

\begin{tabular}{|c|c|c|c|c|c|c|c|c|c|}
\hline Galaxy & Type $^{a}$ & $\mathrm{D}(\mathrm{Mpc})^{b}$ & $\mathrm{M}_{B}^{3}$ & $\mathrm{i}\left({ }^{\circ}\right)$ & $\begin{array}{c}\text { p.a. } \\
\text { major axis }\end{array}$ & $\begin{array}{l}\text { p.a. } \\
\text { bar }\end{array}$ & $\begin{array}{r}\text { scale } \\
(\mathrm{pc} / \operatorname{arcsec})\end{array}$ & $\begin{array}{l}\text { Ring } a^{d} \\
(\operatorname{arcsec})\end{array}$ & $\begin{array}{l}\text { Ring } b^{d} \\
(\operatorname{arcsec})\end{array}$ \\
\hline NGC1097 & $\mathrm{SB}(\mathrm{rs}) \mathrm{bc}$ & $16.8^{e}$ & -20.97 & $46 \pm 5^{6}$ & $134 \pm 3^{6}$ & $139^{g}$ & 81.4 & 19.4 & 16.6 \\
\hline NGC1672 & $\mathrm{SB}(\mathrm{s}) \mathrm{b}$ & $15.1^{h}$ & -19.86 & $36^{i}$ & $152^{i}$ & $94^{i}$ & 73.1 & 11 & 9 \\
\hline \multirow[t]{2}{*}{ NGC5248 } & $\mathrm{SAB}(\mathrm{rs}) \mathrm{bc}$ & $14.0^{h}$ & -19.93 & $61^{j}$ & $146^{j}$ & no $b^{k}{ }^{k}$ & 67.8 & 17.1 & 10.6 \\
\hline & & & & $50-60^{l}$ & $113^{l}$ & & & & \\
\hline
\end{tabular}

a De Vaucouleurs et al. 1976(RC2).

b $H_{0}=75 \mathrm{~km} \mathrm{~s}^{-1} \mathrm{Mpc}^{-1}$.

c $B_{T}$ from Sandage \& Tammann 1981.

d Major (a) and minor (b) axis ring diameters from Buta \& Crocker 1993 for NGC 1097 and 5248 and from the present paper for NGC 1672.

e Hummel, van der Hulst, \& Keel 1987.

f Ondrechen, van der Hulst, \& Hummel 1989.

g Blackman 1981.

h Using radial velocity from Sandage \& Tammann 1981.

i Baumgart \& Peterson 1986.

j Garcia-Gomez \& Athanassoula 1991.

k This work.

1 Corradi \& Capaccioli 1991.

TABLE 2

LOG OF OBSERVATIONS ${ }^{\mathrm{a}}$

\begin{tabular}{lrrrr}
\hline \hline GALAXY & $\begin{array}{r}\text { FILTER } \\
(\AA / \Delta \AA)\end{array}$ & $\begin{array}{r}\text { EXPOSURE } \\
\text { (seconds) }\end{array}$ & $\begin{array}{r}\text { SCALE } \\
\text { arcsec pix }\end{array}$
\end{tabular}

amages from $1.5 \mathrm{~m}$ telescope.

TABLE 3

Log of SPECTRAL ObSERvations ${ }^{\mathrm{a}}$

\begin{tabular}{|c|c|c|c|c|c|c|}
\hline GALAXY & $\begin{array}{r}\text { RANGE } \\
(\AA)\end{array}$ & $\begin{array}{r}\text { P.A. } \\
\text { (degrees) }\end{array}$ & $\begin{array}{r}\text { EXPOSURE } \\
\text { (seconds) }\end{array}$ & $\begin{array}{r}\text { SCALE } \\
\text { arcsec pix }\end{array}$ & DATE & COMMENTS \\
\hline \multirow[t]{10}{*}{ NGC1097 } & $6200-7200$ & 130 & 2700 & 0.8 & 1991 Nov. $1 / 2$ & through nucleus \\
\hline & $6200-7200$ & 130 & 2700 & 0.8 & 1991 Nov. $1 / 2$ & $8^{\prime \prime} \mathrm{NE}$ of nucleus \\
\hline & $6200-7200$ & 130 & 2700 & 0.8 & 1991 Nov. $1 / 2$ & $8^{\prime \prime} \mathrm{SW}$ of nucleus \\
\hline & $6200-7200$ & 40 & 1800 & 0.8 & 1991 Nov. $1 / 2$ & through nucleus \\
\hline & $6200-7200$ & 40 & 1800 & 0.8 & 1991 Nov. $1 / 2$ & $9^{\prime \prime} \mathrm{NW}$ of nucleus \\
\hline & $6200-7200$ & 40 & 1800 & 0.8 & 1991 Nov. $1 / 2$ & $9^{\prime \prime} \mathrm{SE}$ of nucleus \\
\hline & $3400-7000$ & 130 & 685 & 0.9 & 1992 Oct. $4 / 5$ & through nucleus \\
\hline & $3400-7000$ & 170 & 600 & 0.9 & 1992 Nov. $24 / 25$ & through nucleus \\
\hline & $3700-6800$ & 139 & 1800 & 0.8 & 1994 Jan. $6 / 7$ & through nucleus \\
\hline & $6200-7200$ & 12.5 & 1800 & 0.8 & 1994 Jan. $4 / 5$ & through nucleus \\
\hline \multirow[t]{9}{*}{ NGC1672 } & $6200-7200$ & 170 & 1800 & 0.8 & 1991 Nov. $2 / 3$ & through nucleus \\
\hline & $6200-7200$ & 130 & 1200 & 0.8 & 1991 Nov. $2 / 3$ & through nucleus \\
\hline & $6200-7200$ & 130 & 1200 & 0.8 & 1991 Nov. $2 / 3$ & $6^{\prime \prime} \mathrm{SW}$ of nucleus - poor seeing \\
\hline & $6200-7200$ & 130 & 1200 & 0.8 & 1991 Nov. $2 / 3$ & $10^{\prime \prime} \mathrm{NE}$ of nucleus \\
\hline & $6200-7200$ & 130 & 1200 & 0.8 & 1991 Nov. $2 / 3$ & $5^{\prime \prime} \mathrm{NE}$ of nucleus - poor seeing \\
\hline & $3400-7000$ & 130 & 600 & 0.9 & 1992 Nov. $24 / 25$ & through nucleus \\
\hline & $3700-6800$ & 94 & 1800 & 0.8 & 1994 Jan. $5 / 6$ & through nucleus \\
\hline & $6200-7000$ & 152 & 1800 & 0.8 & 1994 Jan. $4 / 5$ & through nucleus \\
\hline & $6200-7000$ & 170 & 600 & 0.8 & 1994 Jan. $4 / 5$ & through nucleus \\
\hline \multirow[t]{5}{*}{ NGC5248 } & $6000-7000$ & 146 & 1800 & 0.9 & 1992 May $29 / 30$ & through nucleus \\
\hline & $6000-7000$ & 146 & 1800 & 0.9 & 1992 May $29 / 30$ & $3.4^{\prime \prime} \mathrm{NE}$ of nucleus \\
\hline & $6000-7000$ & 146 & 1800 & 0.9 & 1992 May $29 / 30$ & $4.3^{\prime \prime} \mathrm{SW}$ of nucleus \\
\hline & $3400-7000$ & 146 & 1800 & 0.9 & 1992 May $28 / 29$ & through nucleus \\
\hline & $3400-7000$ & 146 & 1800 & 0.9 & 1992 May $28 / 29$ & $3.4^{\prime \prime} \mathrm{NE}$ of nucleus \\
\hline
\end{tabular}

a From $4 \mathrm{~m}$ telescope. 
Under these circumstances, differential refraction can cause errors in relative fluxes for lines widely separated in wavelength. This effect is only significant for [O II] 23727 . The component of differential refraction perpendicular to the slit between [O II] $\lambda 3727$ and [S II] $\lambda 6724$ is $<0$ ".4 in four of the seven low-dispersion long-slit spectra, and errors from this effect are negligible. For the other three low-dispersion spectra, this component of differential refraction is 0.8 (NGC 1097, P.A. $170^{\circ}$ ), 1".1 (NGC 1672, P.A. 130 ${ }^{\circ}$ ), and 0".6 (NGC 1672 , P.A. $94^{\circ}$ ). In these cases, the resulting error in the flux ratio [O II] $\lambda 3727 /[\mathrm{S} \mathrm{II}] \lambda 6724$ may be up to a few tens of percent, depending on the precise brightness distribution of the nebulosity. Between [O II] $\lambda 3727$ and [O III] 25007 , the largest component of differential refraction perpendicular to the slit is 0 .'7, which will give an error of up to a few tens of percent in the $[\mathrm{O} \mathrm{II}] /[\mathrm{O} \mathrm{III}]$ flux ratio. The component of differential refraction along the slit was eliminated in the reductions by tracing the curve of the nuclear spectrum in the long-slit "image" and using the same form for off-nuclear positions.

\section{RESULTS}

\subsection{Images}

\subsection{1. $N G C 1097$}

The red continuum $(7099 \AA)$ image of NGC 1097 is shown in Figure $1 a$, where the bar can be observed extending $\approx 90^{\prime \prime}$ to the NW and SE. The position angle of the bar, as determined from this image, is $141^{\circ} \pm 2^{\circ}$, in agreement with the value of $139^{\circ}$ obtained by Blackman (1981). This

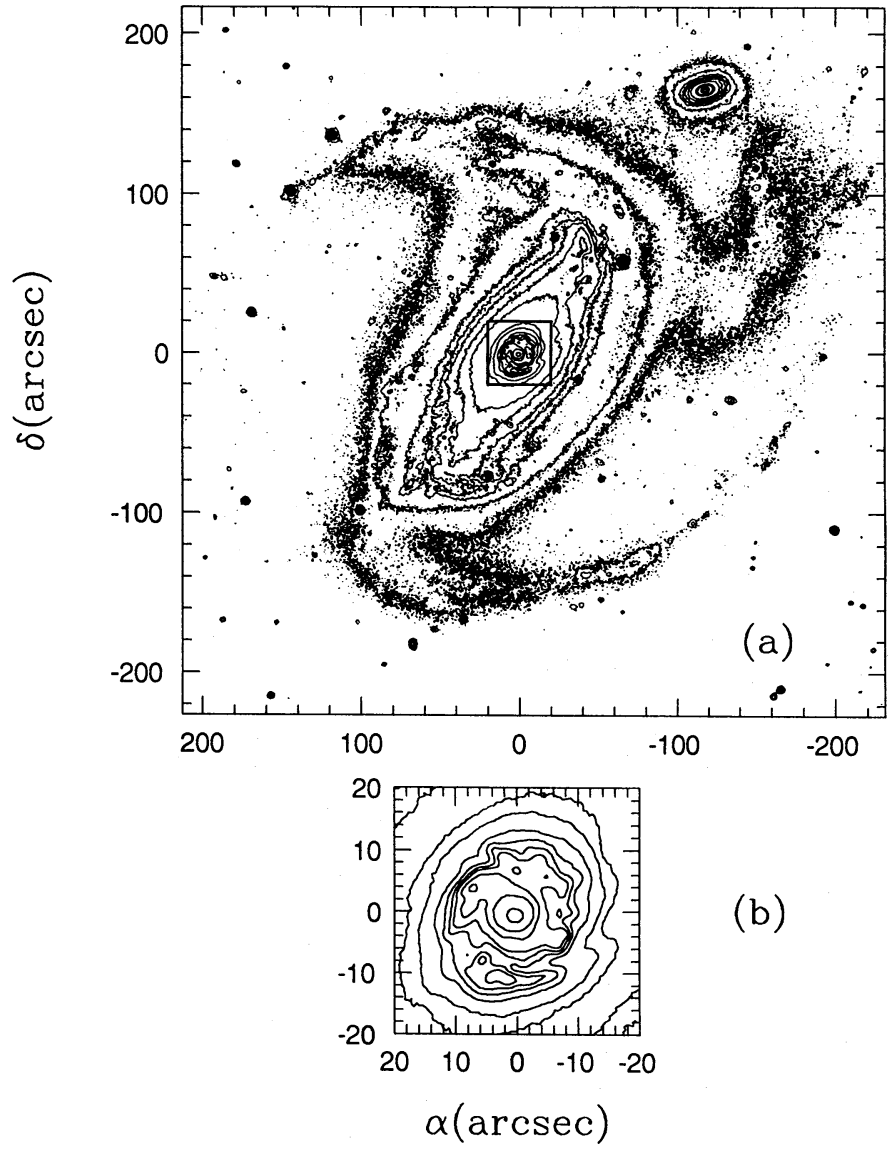

image (Fig. 1b) shows that the morphology of the nuclear ring in continuum light is more of a tight spiral than of a ring, in agreement with the results of Forbes et al. (1992) from near-IR images. In Figure $1 c$ we show the continuum subtracted $\mathrm{H} \alpha+[\mathrm{N}$ II $]$ image, which maps the emission of the gas. Our image agrees well with that presented by Hummel et al. (1987). It can be seen (see Fig. 1d) that the gaseous nuclear ring has an oval shape, although it is patchy and somewhat distorted. The largest and smallest outer radii of this ring, measured in the $\mathrm{H} \alpha$ image, are $16^{\prime \prime}$ at P.A. $\approx 13^{\circ}$, and $\approx 12^{\prime \prime}$ along the approximately perpendicular direction, respectively. There is faint line emission between the compact nuclear source and the ring. Figure $1 c$ also shows $\mathrm{H}$ II regions in the bar and spiral arms. The dwarf elliptical companion to NGC 1097-NGC 1097Acan be seen at the NW corner of the red continuum image but disappears in the continuum-subtracted image, confirming an absence of line emission and indicating that the continuum was well subtracted.

\subsection{2. $N G C 1672$}

We show the red continuum image of NGC 1672 in Figures $2 a-2 b$, where the bar can be observed extending to $\approx 70^{\prime \prime}$ to the east and west, at P.A. $=97^{\circ} \pm 3^{\circ}$, in agreement with the value of $94^{\circ}$ from Baumgart \& Peterson (1986). Several dust lanes are found along the bar. The morphology of the nuclear region in the continuum shows a knotty structure, although much smoother than that observed in the $\mathrm{H} \alpha$ image. This latter image is shown in Figs. $2 c-2 d$, where hot spots are seen in the nuclear ring, and many $\mathrm{H}$ II

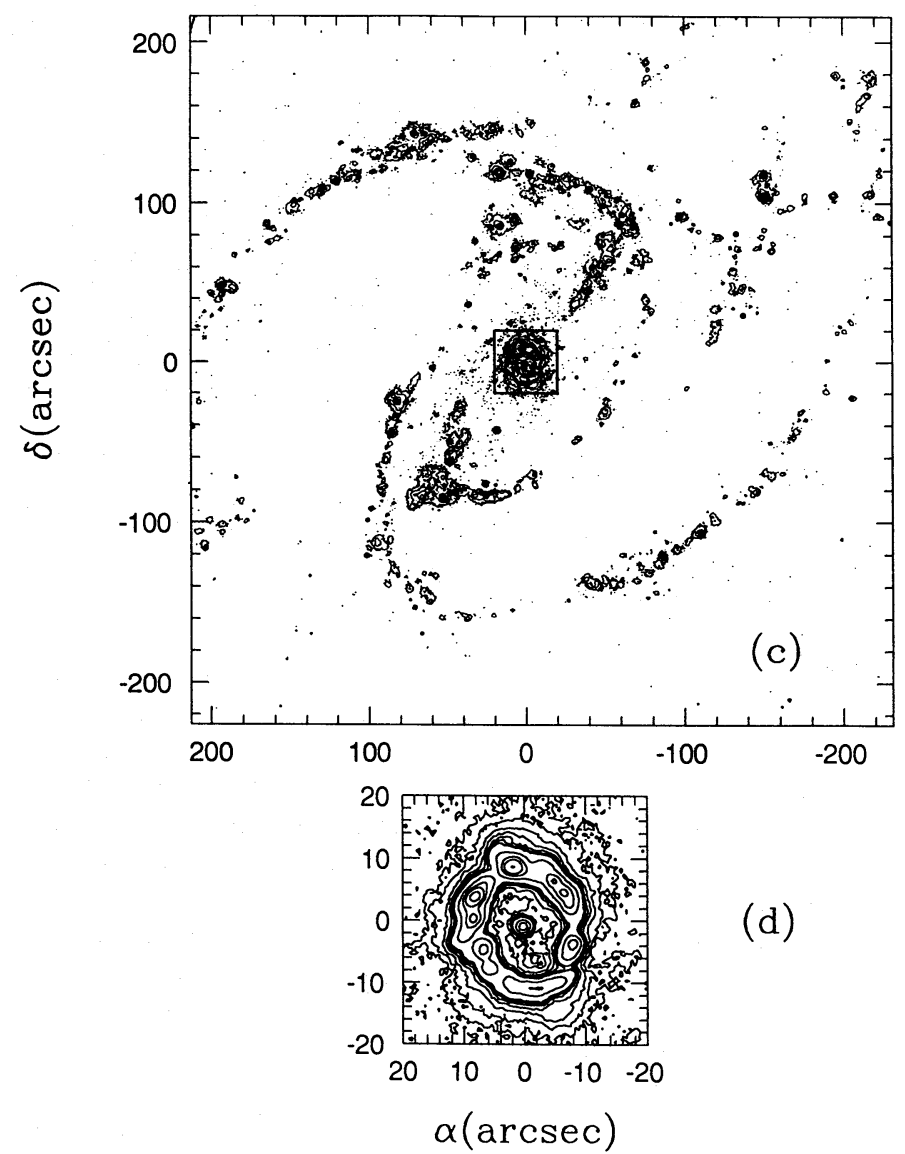

Fig. 1.- $(a-b)$ Contour maps of the red continuum (7099 $\AA$ ) image of NGC 1097 , with levels at $1,2.2,2.7,3,3.3,4,5,8,10,15,20,25,30,40,50$, and $80 \%$ of the peak brightness above the sky. (c-d) Continuum-subtracted $\mathrm{H} \alpha$ image, with levels at $0.7,1.5,3,5,7,8.4,10,20,35,49$, and $79 \%$ of peak brightness above the sky. Panels $b$ and $d$ show enlarged versions of the region indicated by the square in panels $a$ and $c$. 

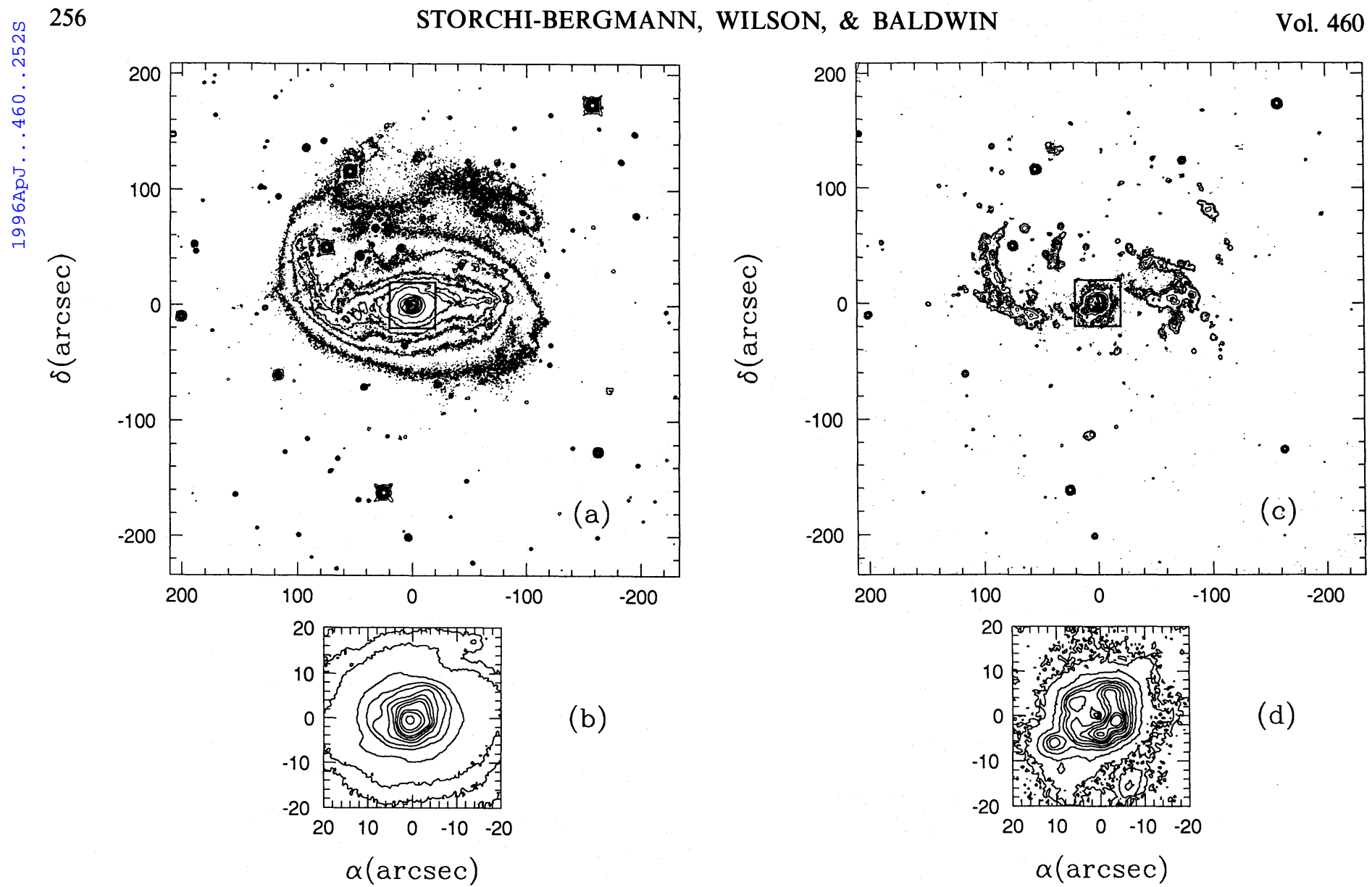

Fig. 2.- $(a-b)$ Contour maps of the red continuum (7099 $\AA$ ) image of NGC 1672 , with levels at $1.2,1.5,1.7,2,2.5,3,5,7.5,10,15,20,25,30,40,50$, and $80 \%$ of the peak brightness above the sky. $(c-d)$ Continuum-subtracted $\mathrm{H} \alpha$ image, with levels at $0.6,2.4,3.5,4,6,10,15,20,30,40,50,65$, and $80 \%$ of peak brightness above the sky. Panels $b$ and $d$ show enlarged versions of the region indicated by the square in panels $a$ and $c$.

regions are visible along the bar and in the spiral arms. The ring, seen most clearly in the $\mathrm{H} \alpha$ image, is slightly elongated along P.A. $\approx 130^{\circ}$, with outer major and minor axis radii of $\approx 11^{\prime \prime}$ and $9^{\prime \prime}$, respectively. The ring seems not to be centered at the nucleus (assumed to coincide with the peak of the continuum light), but at $\approx 1^{\prime \prime}-2^{\prime \prime}$ to the NE. A knot of emission is observed to the SE, just outside the ring, and roughly along the same position angle as the major axis of the ring. Unlike NGC 1097, the $\mathrm{H} \alpha$ emission of the nucleus NGC 1672 is very weak - the isophotal map has a minimum close to the continuum nucleus.

\subsection{3. $N G C 5248$}

Figures $3 a-3 b$ show the continuum image of NGC 5248 . Its classification as SAB is probably a result of the elongated (in the E-W direction) appearance of the inner 30" region. Nevertheless, careful inspection of the continuum and $\mathrm{H} \alpha$ (Figs. $3 c-3 d$ ) images using the television display suggests that the spiral arms begin near the outer edge of the nuclear ring and that there is no clear evidence for a bar. The ring shows several knots in the continuum image (Fig. $3 b$ ). The $\mathrm{H} \alpha$ image of the ring (Fig. $3 d$ ) shows two large blobs to NW and SE and one smaller blob to the south of the nucleus, in agreement with a previous image by Pogge (1989). The outer radii of the ring are $\approx 11^{\prime \prime}$ along P.A. $\approx 120^{\circ}$ and $\approx 6^{\prime \prime}$ along the perpendicular direction. The $\mathrm{H} \alpha$ emission at the location of the continuum nucleus is very weak, as was found for NGC 1672. Several H II regions can be seen along the spiral arms.

\subsection{Spectroscopy}

In the nuclear region, spectra were extracted by binning together 2 pixels along the slit for the 1991 and 1992 data, and 2.5 pixels for the 1994 data. The resolutions are thus $2^{\prime \prime} \times 11^{\prime \prime} 6$ (1991), $2^{\prime \prime} \times 11^{\prime \prime} 8$ (1992), and $2^{\prime \prime} \times 2^{\prime \prime}(1994)$. Far from the nucleus, more pixels were binned together in order to improve the $\mathrm{S} / \mathrm{N}$ ratio; in the following analysis, we only use spectra with a flux in the [S II] $\lambda \lambda 6717,31$ lines $>10^{-16}$ ergs $\mathrm{cm}^{-2} \mathrm{~s}^{-1}$ for the spectra with $1800 \mathrm{~s}$ integration time (or longer), and $>2 \times 10^{-16} \mathrm{ergs} \mathrm{cm}^{-2} \mathrm{~s}^{-1}$ for the spectra with $600 \mathrm{~s}$ integration times, which correspond to $\approx 3-5 \sigma$ detection. A sample of spectra at representative locations in each galaxy (nucleus, ring, and an external $\mathrm{H}$ il region for all the three galaxies, plus a location between the nucleus and the ring and a typical disk location for NGC 1097) are shown in Figures 4, 5, and 6.

\section{GASEOUS KINEMATICS}

In order to obtain the gaseous velocity field, we have measured the wavelengths of the peaks of the $\mathrm{H} \alpha,[\mathrm{N}$ II] $\lambda \lambda 6548,6584$ and [S II] emission lines in the $1.7 \AA$ resolution spectra. We did not find any differences between the radial velocities obtained from the different lines and decided to adopt the $\mathrm{H} \alpha$ velocity as representative of the velocity field of the galaxy.

We show in Figure 7 the continuum-subtracted $\mathrm{H} \alpha$ image of NGC 1097 with a representation of the velocity field superposed, after subtraction of the systemic velocity (see 


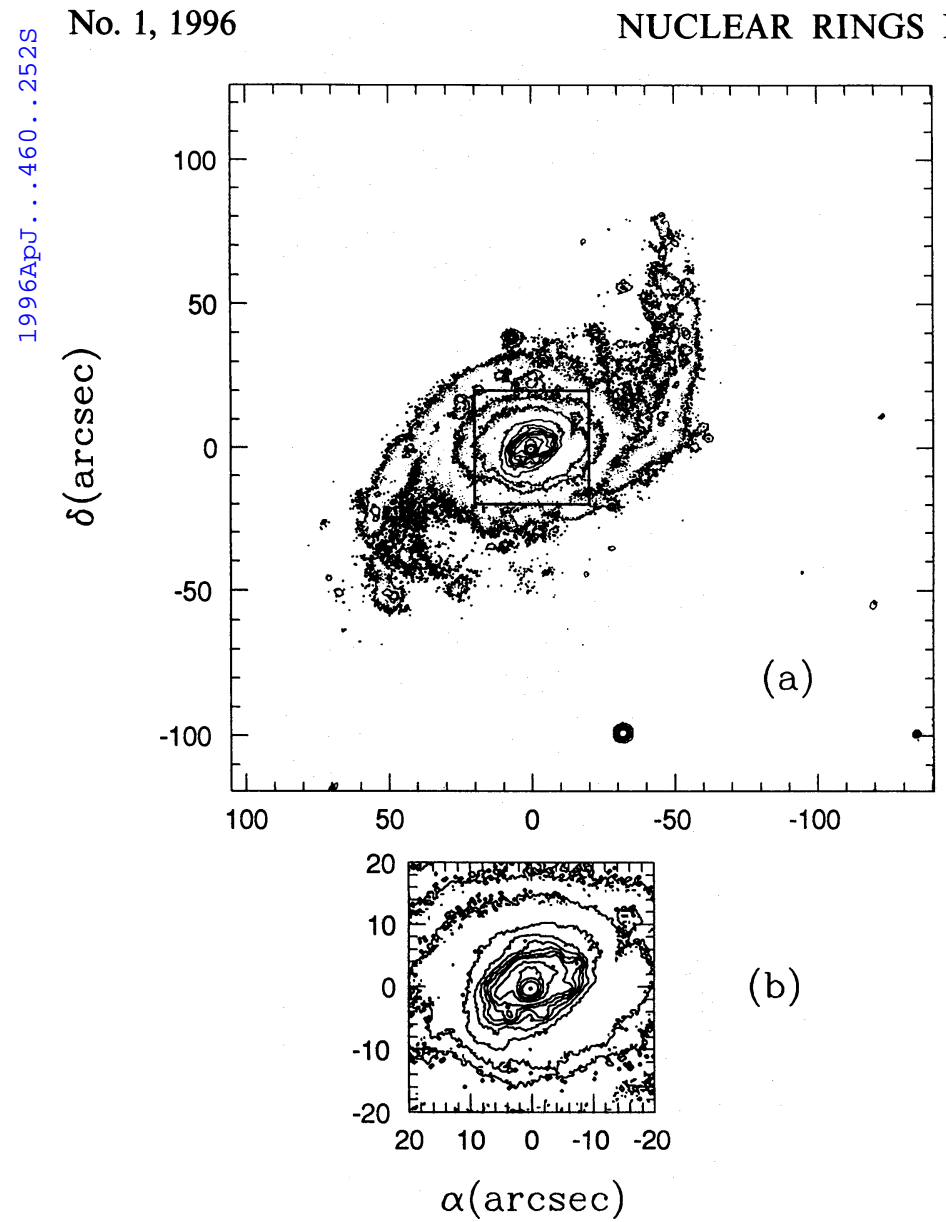

Fig. 3.- $(a-b)$ Contour maps of the red continuum (7099 $\AA$ ) image of NGC 5248, with levels at 4, 6.6, 10, 13, 16.6, 20, 23, 26.5, 33, 40, 46.4, 60, 80, and $100 \%$ of the peak brightness above the sky. (c-d) Continuum-subtracted $\mathrm{H} \alpha$ image, with levels at $1,2.7,8.1,21.6,27,40,50$, and $80 \%$ of peak brightness above the sky. Panels $b$ and $d$ show enlarged versions of the region indicated by the square in panels $a$ and $c$.

below and Table 4). The circles represent the velocity of the peak of $\mathrm{H} \alpha$ emission at each location, with the size of the circles being proportional to the magnitude of the velocity, open symbols representing negative velocities (blueshifts), and filled symbols positive velocities (redshifts). Figures 8 and 9 are similar plots of the velocity fields of NGC 1672 and NGC 5248, respectively.

In order to obtain the kinematic line of nodes and the inclination of each galaxy, we have fitted a simple functional form to the data, assuming that the gas follows circular orbits in a plane (Bertola et al. 1991):

$$
V_{\text {rot }}(r)=V_{\text {sys }}+\frac{A r}{\left(r^{2}+C_{0}^{2}\right)^{p / 2}}
$$

where $V_{\text {sys }}$ is the systemic velocity, $A, C_{0}$, and $p$ are parameters, and $r$ is the radius in the plane of the galaxy. The observed radial velocity at a position $(R, \Psi)$, where $R$ is the projected radial distance from the nucleus in the plane of the sky and $\Psi$ is the corresponding position angle, is given by

$$
\begin{aligned}
& V_{\text {mod }}(R, \Psi) \\
& =V_{\text {sys }}+\frac{A R \cos \left(\Psi-\Psi_{0}\right) \sin i \cos ^{p} i}{\left\{R ^ { 2 } \left[\sin ^{2}\left(\Psi-\Psi_{0}\right)\right.\right.} \\
& \left.\left.\quad+\cos ^{2} i \cos ^{2}\left(\Psi-\Psi_{0}\right)\right]+C_{0}^{2} \cos ^{2} i\right\}^{p / 2}
\end{aligned}
$$

where $i$ is the inclination of the disk ( $i=0$ for a face on disk) and $\Psi_{0}$ is the position angle of the line of nodes. We determined the various parameters simultaneously by minimizing the residuals $\Delta V=V_{\text {obs }}-V_{\text {mod }}$, where $V_{\text {obs }}(R, \Psi)$ is the observed radial velocity at position $(R, \Psi)$ in the plane of the sky. We fitted the model to the observed velocity field by means of a nonlinear least-squares (Levenberg-Marquardt) algorithm, in which initial guesses must be given for the parameters.

When we allowed all parameters to be free, the method converged to reasonable values only for NGC 5248. For

TABLE 4

Kinematic Parameters

\begin{tabular}{lrrrrrr}
\hline \hline GALAXY & $V_{\text {sys }}\left(\mathrm{km} \mathrm{s}^{-1}\right)$ & $\Psi_{o}\left({ }^{\circ}\right)$ & $i\left(^{\circ}\right)$ & $A\left(\mathrm{~km} \mathrm{~s}^{-1}\right)$ & $C_{o}(\mathrm{kpc})$ & $p$ \\
\hline NGC1097 & $1271 \pm 15$ & $135 \pm 2$ & $46^{a}$ & $308 \pm 8$ & $0.23 \pm 0.04$ & $1.18 \pm 0.02$ \\
NGC1672 & $1337 \pm 13$ & $121 \pm 3$ & $36^{a}$ & $140 \pm 4$ & $0.07 \pm 0.03$ & $1.02 \pm 0.02$ \\
NGC5248 & $1183 \pm 16$ & $119 \pm 6$ & $45 \pm 5$ & $138 \pm 10$ & $0.20 \pm 0.11$ & $1.05 \pm 0.04$ \\
\hline
\end{tabular}

${ }^{\text {a }}$ Adopted. 


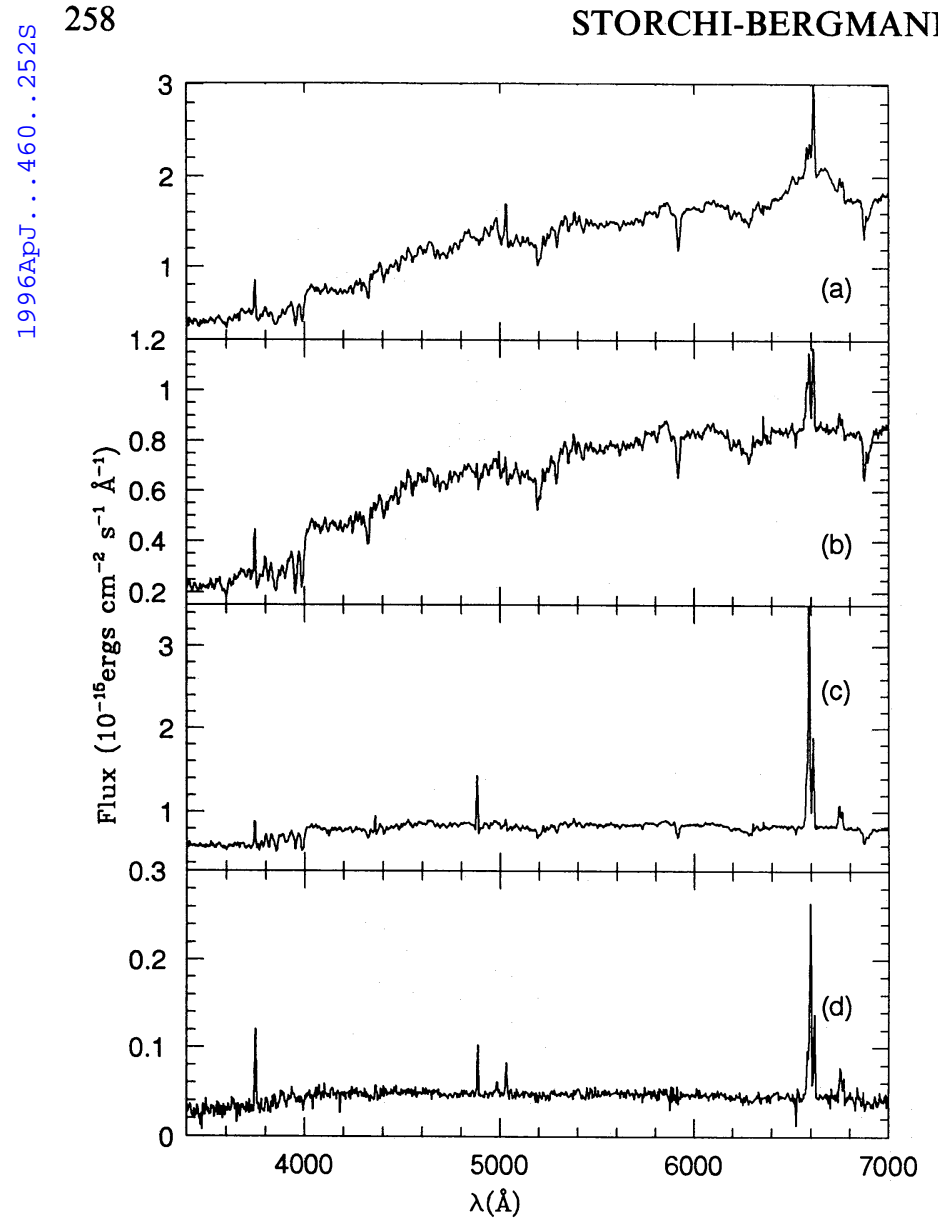

Vol. 460

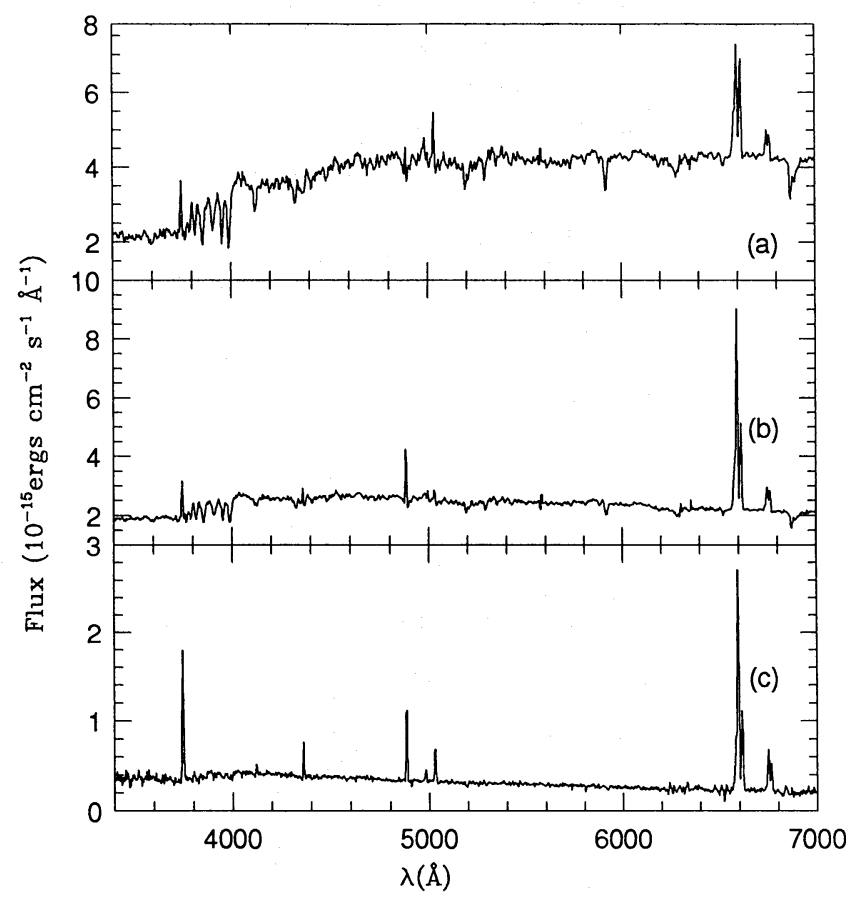

Fig. 5.-Representative spectra of different regions in NGC 1672: (a) nucleus, (b) ring, (c) $\mathrm{H}$ il region at $52^{\prime \prime} \mathrm{NW}$ of the nucleus.

agreement with the maximum rotation velocity of Ondrechen et al. For NGC 1672, our kinematic line of nodes is $\approx 30^{\circ}$ smaller than the photometric value obtained by Baumgart \& Peterson (1986) from near-infrared imaging. For NGC 5248, our major axis position angle and inclination agree well with the values listed by Corradi \& Capaccioli (1991), based on data from Burbidge, Burbidge, \& Prendergast (1962). However, our kinematic line of notes is $27^{\circ}$ smaller than the more recent determination by GarciaGomez \& Athanassoula (1991), using the distribution of $\mathrm{H}$ II regions.

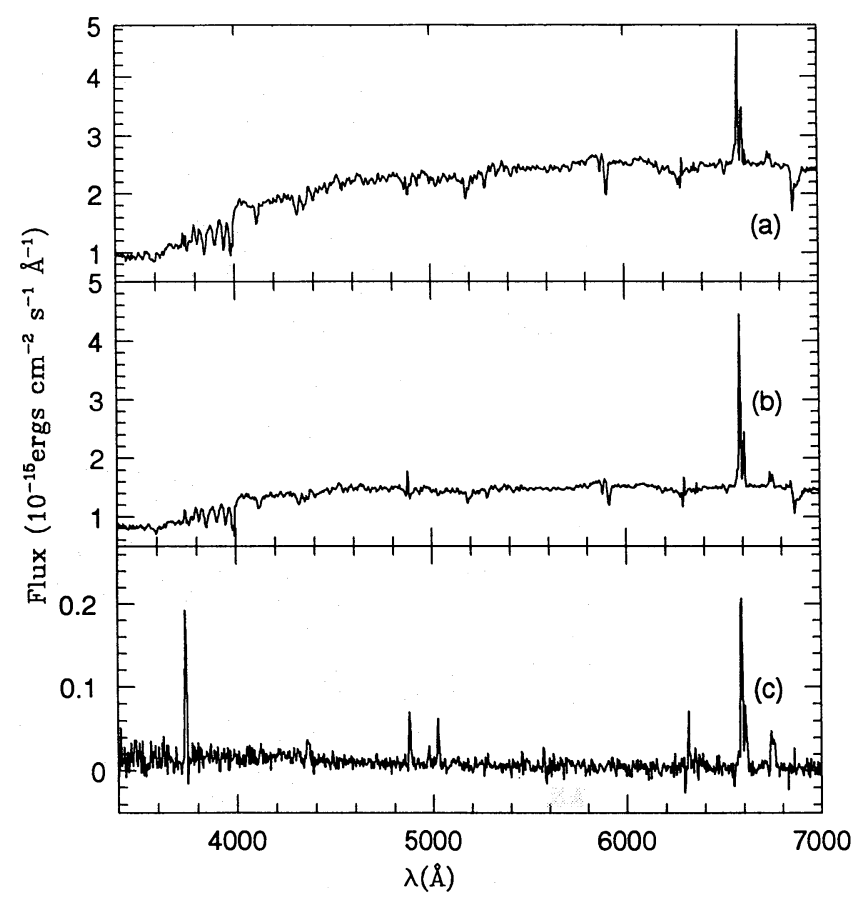

FIG. 6.-Representative spectra of different regions in NGC 5248: (a) nucleus, $(b)$ ring, $(c) \mathrm{H}$ il region at 134 " SE of the nucleus. 


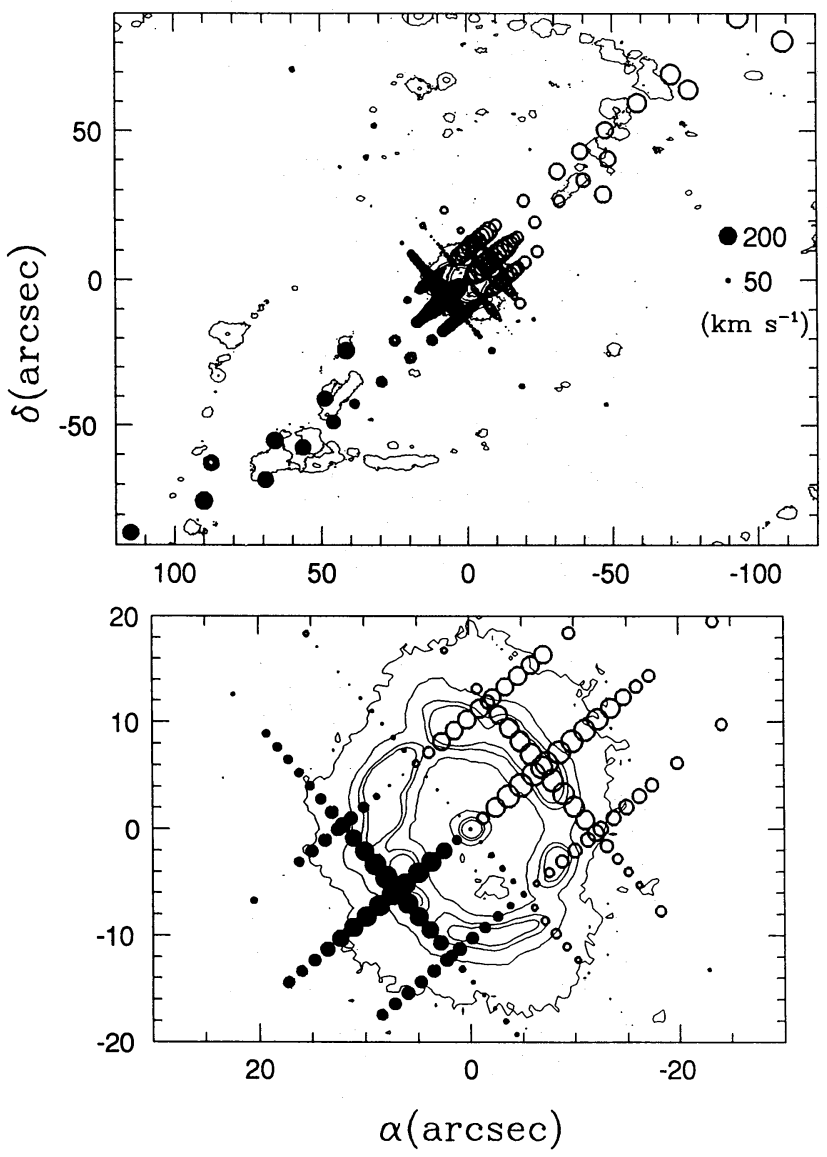

FIG. 7.-Top: circles represent the peak $\mathrm{H} \alpha$ velocities (after subtraction of the systemic velocity) superimposed on the continuum subtracted $\mathrm{H} \alpha$ image of NGC 1097, with the radii of the circles being proportional to the magnitude of the velocity. Open symbols represent blueshifts, and filled symbols represent redshifts. Bottom: the nuclear region is shown enlarged.

The behavior of the rotation curves and quality of the fits can be seen in Figure 10, where we have plotted the velocities obtained from the long-slit observations through the nuclei along the position angles closest to the kinematic line of nodes, together with the models. These position angles are $130^{\circ}, 130^{\circ}$, and $146^{\circ}$, for NGC 1097 , NGC 1672 , and NGC 5248, respectively.

\subsection{Discussion}

From the observed velocity field and assuming that the spiral arms are trailing, we find that the near side of the galactic disk is to the SW for NGC 1097 and NGC 5248, and to the NE for NGC 1672.

In the model describing the rotation curves, $C_{0}$ (see Table 4) is the radius at which the rotational velocity reaches $\approx 70 \%$ of $V_{\max }$. Assuming that Figure 6 of Rubin et al. (1985) represent typical rotation curves, it can be seen that the value of $C_{0}$ for types $\mathrm{Sb}$ and $\mathrm{Sc}$ is $\approx 2 \mathrm{kpc}$, while for our sample the largest value is $0.21 \mathrm{kpc}$, which suggests that the three galaxies have anomalously large central velocity gradients. Nevertheless, inspection of the individual rotation curves presented by Rubin et al. (1985) in their Appendix shows that four of the $23 \mathrm{Sb}$ galaxies and other four of the $21 \mathrm{Sc}$ galaxies in their sample reach $70 \%$ of $V_{\max }$ at a distance of $0.5 \mathrm{kpc}$ from the nucleus (there is no information for radii between 0 and $0.5 \mathrm{kpc}$ ). It is interesting to note that one of these Sc galaxies is NGC 4321, which also has a nuclear ring (Buta \& Crocker 1993).
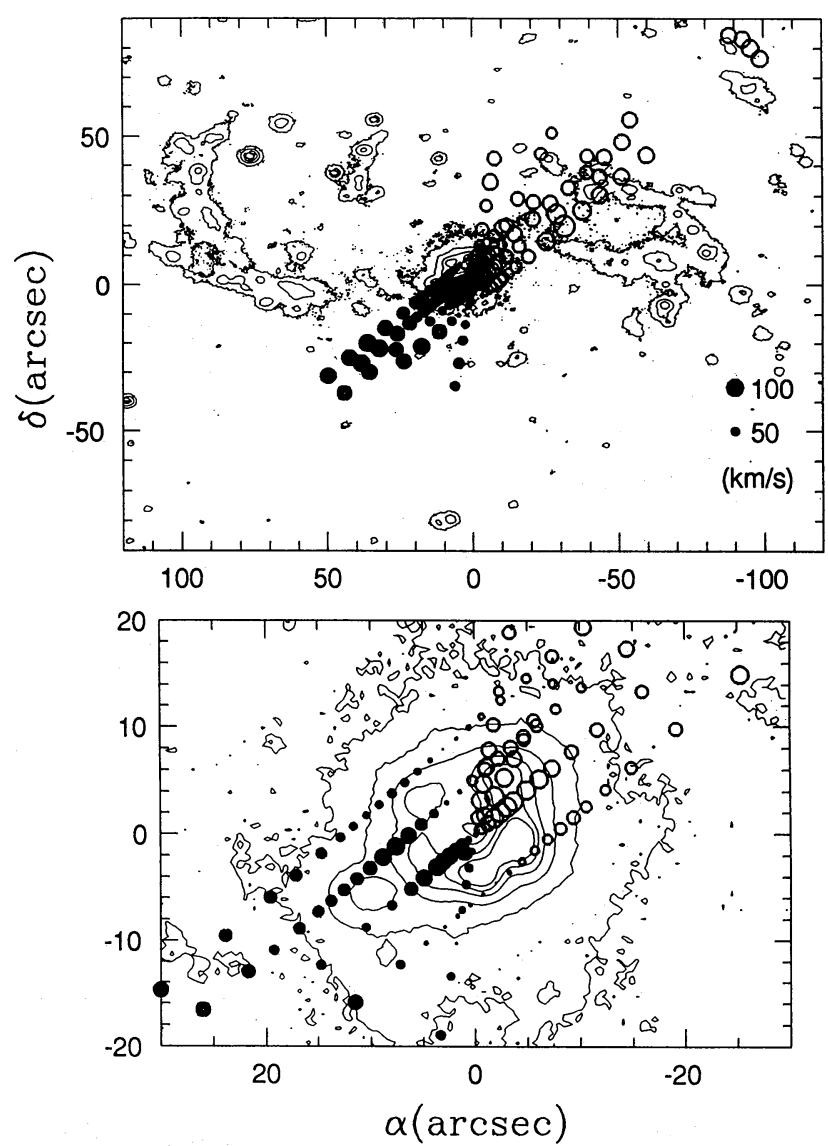

Fig. 8. - The velocity field of NGC 1672, in the same format as Fig. 7
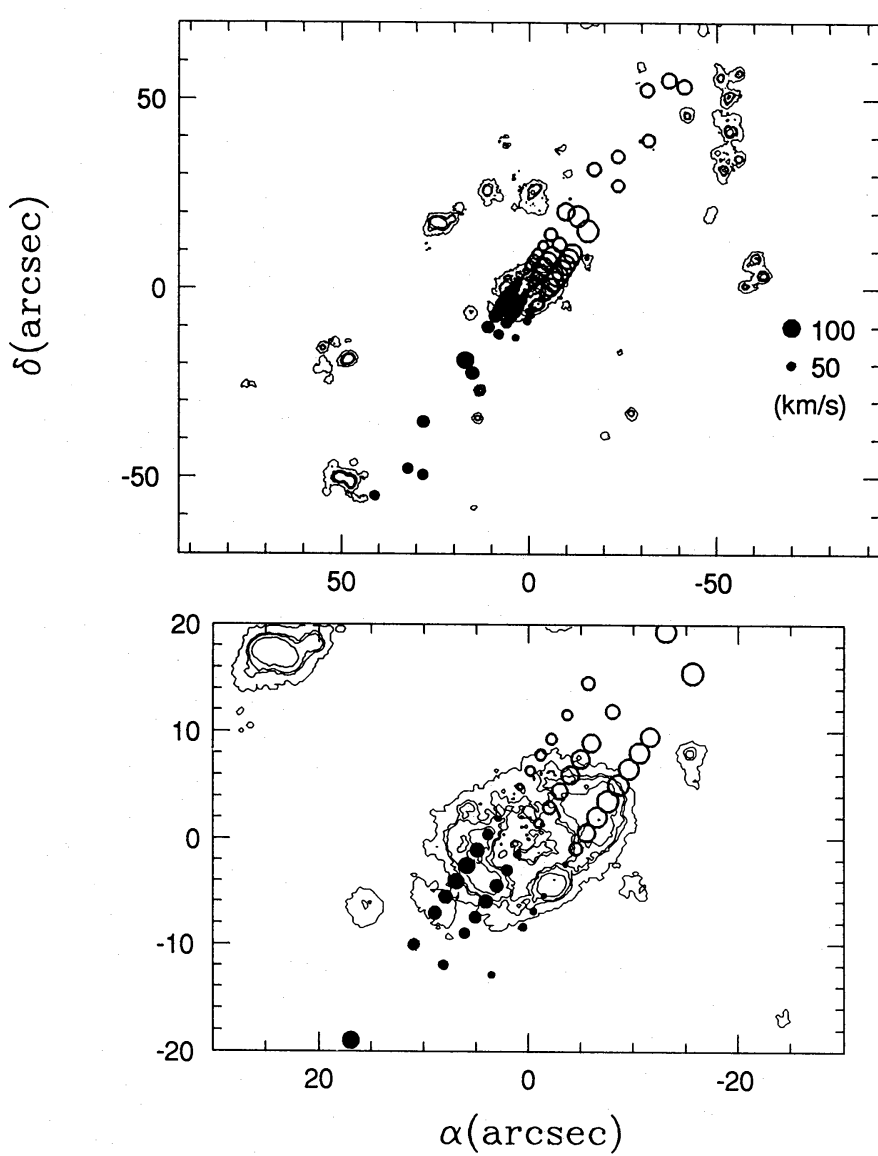

Fig. 9.--The velocity field of NGC 5248, in the same format as Fig. 7 


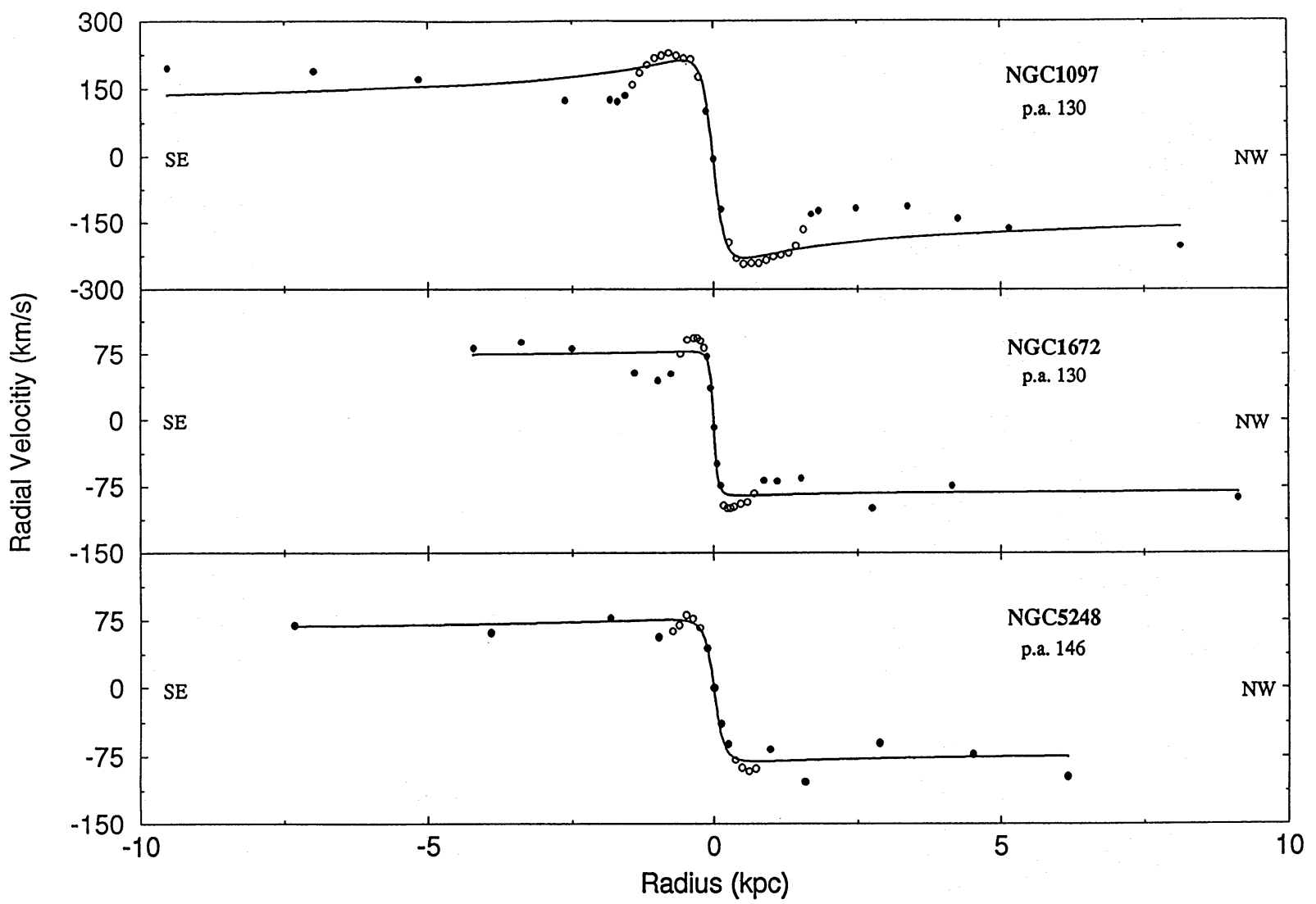

FIG. 10.-Rotation curve along the position angle closest to the kinematic line of nodes for the three galaxies, together with the best model (from fits to data points in all position angles). Open symbols identify data points from the rings.

One tentative conclusion from the above results is that the rings appear in galaxies with large central concentrations of mass. This is indeed one of the conclusions reached by Piner et al. (1995) in recent numerical hydrodynamic simulations of the gas dynamics in barred galaxies. They show that nuclear gas rings form as a result of the potential of the bar, which drives the gas in toward the center. Piner et al. (1995) find only nuclear rings in galaxies with two ILRs, and, in all cases, the ring is situated between the two ILRs. The two ILRs occur only for high central mass concentrations (above $6 \times 10^{9} M_{\odot} \mathrm{kpc}^{-3}$ for the standard model of Piner et al. 1995). Piner et al. (1995) also follow the evolution of the ring, showing that initially it is elliptical and elongated along the bar minor axis, but after $\approx 1.2 \mathrm{Gyr}$ (for their standard model) becomes approximately circular. During this time, the ring also moves inward and becomes narrower, denser, and more massive. There are azimuthal density variations within the ring of factors of 3 , with the highest densities occurring along the bar major axis.

In order to compare the predicted and observed characteristics of the ring, we now derive the intrinsic morphology of the rings, under the assumption that they are in the plane of the galaxy disk. From the major axis position angle and the inclination, we obtain, for NGC 1097, an outer semimajor axis of $1.66 \mathrm{kpc}$ and an outer semiminor axis of 1.10 kpc. For NGC 1672, the semimajor and minor axes are 0.85 and $0.80 \mathrm{kpc}$, respectively, while for NGC 5248, they are 0.73 and $0.69 \mathrm{kpc}$. The ring in NGC 1097 is elongated approximately perpendicular to the bar, while those in NGC 1672 and 5248 are almost round. These observed characteristics are consistent with the predictions of the model discussed above and suggest that the ring in NGC
1097 may have formed more recently than in the other two galaxies, where the rings are already circular.

In order to determine whether the rings are located between the two ILRS, we have obtained the angular velocities, $\Omega$, and the Lindblad curves, $\Omega \pm \kappa / 2$, from the models fitted to the kinematic data. We point out that there are large uncertainties in these curves (Fig. 11), due to observational errors, the small angular radii of the rotation curve turnovers (Figs. 7-9), the possibility of noncircular motions (see below), and the idealized nature of the model (eq. [1]) used to describe the velocity measurements. To fix the pattern speed, $\Omega_{p}$, we have assumed that corotation lies at the end of the bar for NGC 1097 and NGC 1672 (e.g., Sparke \& Sellwood 1987). The locations of the ILRs can then be obtained as the intersections between the $\Omega_{p}$ and $\Omega-\kappa / 2$ curves (see Fig. 11). Inspection of Figure 11 shows that NGC 1097 and 1672-the ones with LINER nucleihave stronger mass concentrations than NGC 5248.

For NGC 1097 the bar ends at a radius of $7.3 \mathrm{kpc}$. Using the kinematical model in Table 4 we obtain $\Omega_{p}=29 \mathrm{~km} \mathrm{~s}^{-1}$ $\mathrm{kpc}^{-1}$. The radii at which $\Omega_{p}=\Omega-\kappa / 2$ are 0.05 and 3.0 kpc. For NGC 1672, the bar ends at $5.1 \mathrm{kpc}, \Omega_{p}=27 \mathrm{~km}$ $\mathrm{s}^{-1} \mathrm{kpc}^{-1}$, and the two radii where $\Omega_{p}=\Omega-\kappa / 2$ are 0.02 and $1.6 \mathrm{kpc}$. Comparing with the ring dimensions given above, we conclude that the rings are indeed located between the two ILRs.

In the case of NGC 1097, Ondrechen, van der Hulst, \& Hummel (1989), have suggested that corotation with the spiral pattern is located farther away $(\approx 1.6$ times the bar radius). If the spiral and bar pattern speeds are identical, $\boldsymbol{\Omega}_{p}$ would be reduced to $17 \mathrm{~km} \mathrm{~s}^{-1} \mathrm{kpc}^{-1}$, and the ring would still lie between the two ILRs. Alternatively, the bar could 


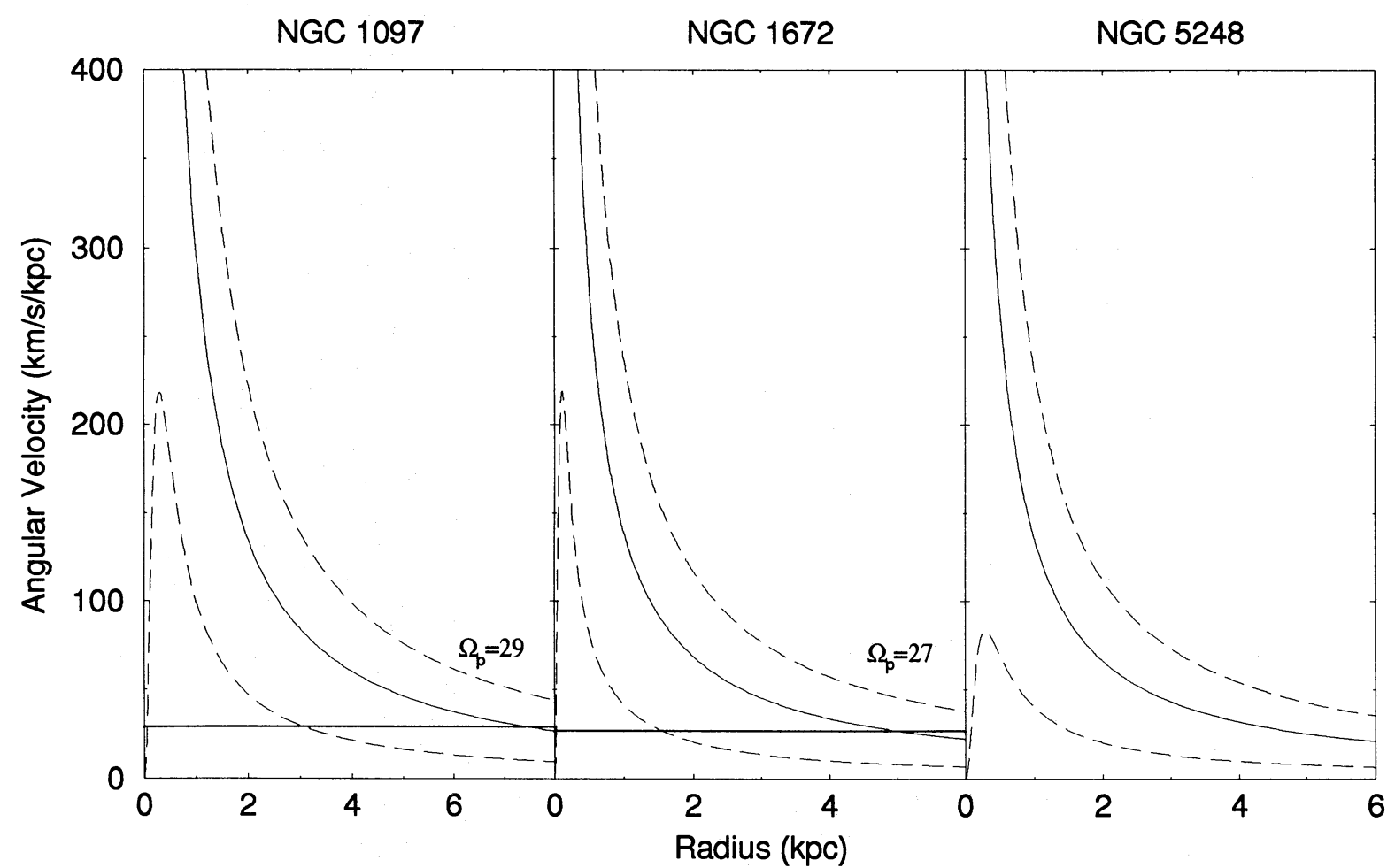

Fig. 11.-Angular velocities $\Omega$ (thin solid lines) and Lindblad curves $\Omega \pm \kappa / 2$ (dashed lines) obtained from the kinematic models fitted to the data. $\Omega_{p}$ (thick solid line) is the pattern speed in the hypothesis that corotation lies at the end of the bar for NGC 1097 and 1672.

rotate faster than the spiral arms, in accordance with results from $N$-body modeling by Sparke \& Sellwood (1987) and Sellwood \& Sparke (1988).

Another feature of all three "rotation curves" is a "hump" at the ring and a sharp dropoff just outside (Fig. 10). These features are most prominent in NGC 1097 and 1672. In the case of NGC 1672, for which the ring is almost round, this decrease may be related to inward motions of the gas driven by the bar. Such motions are expected to occur between corotation and the nuclear ring (e.g., Piner et al. 1995). For NGC 1097, the bar and major axis of the galaxy disk coincide (Table 1), so streaming along the bar should not affect the velocity field along the major axis. Further, the ring is elliptical. In this case, the velocity "hump" at the ring may result from the ring's major axis being perpendicular to the bar. As the gas orbits, it is expected to conserve its angular momentum to first order, so the orbital velocity should be highest when it is closest to the nucleus (i.e., along the bar) and lowest when at its maximum distance from the nucleus (i.e., perpendicular to the bar). Thus, a spectrum taken with the slit along the major axis (Fig. 10) would show that the gas in the ring is orbiting faster than ambient rotation. Yet another possibility is that the gas just outside the ring is not in the plane of the disk and has anomalous kinematics.

\section{EXCITATION}

The medium-resolution spectra were also used to construct maps of emission-line ratios over the central regions of the galaxies. Given the selection of spectra with $>3-5 \sigma$ detection ( $\S 4.2$ ), errors in these ratios are $\leq 20 \%$. Figures 12,13 , and 14 show representations of the spatial variation of the emission-line ratios [N II] $\lambda \lambda 6548,6584 / \mathrm{H} \alpha$ and [S II] $\lambda \lambda 6717,6731 / \mathrm{H} \alpha$ for NGC 1097 , NGC 1672, and NGC 5248 , respectively. It can be seen that the highest $[\mathrm{N} \mathrm{II}] / \mathrm{H} \alpha$ and $[\mathrm{S} \mathrm{III}] / \mathrm{H} \alpha$ ratios in NGC 1097 and NGC 1672 are found at the nucleus and outside the ring. High values of these ratios are found up to $25^{\prime \prime}-50^{\prime \prime}$ from the nucleus. In NGC 1097, the highest ratios for $[\mathrm{N} \mathrm{III}] / \mathrm{H} \alpha$ and $\left[\mathrm{S} \mathrm{II}^{\mathrm{N}} / \mathrm{H} \alpha\right.$ are 2.5 and 0.8 , while in NGC 1672 , they reach 1.5 and 0.7 , respectively. In NGC 5248, $[\mathrm{N} \mathrm{III} / \mathrm{H} \alpha=0.7$ and $[\mathrm{S} \mathrm{II}] / \mathrm{H} \alpha=0.2$ right at the nucleus and reach higher values of $[\mathrm{N} \mathrm{II}] /$ $\mathrm{H} \alpha=0.9$ and $[\mathrm{S} \mathrm{II}] / \mathrm{H} \alpha=0.3$ just outside the ring to the NW. Thus, although presenting a qualitatively similar behavior, the line ratios decrease in the sequence NGC 1097, NGC 1672, NGC 5248. At the ring, in all three galaxies, the $[\mathrm{N} \mathrm{II}] / \mathrm{H} \alpha$ and $\left[\mathrm{S} \mathrm{II}^{\mathrm{I}}\right] / \mathrm{H} \alpha$ ratios are 0.3 and 0.1 , respectively, which are typical of $\mathrm{H}$ II regions.

We also show in Figures 12,13 and 14 the distribution of the FWHM of the [N II] $\lambda 6584$ emission line over the central regions of the galaxies, as well as the gas densities determined from the line ratio [S $\mathrm{S}$ II] $\lambda 6716 / \lambda 6731$ (McCall 1984). In the three galaxies, the FWHM is $\sim 120-150 \mathrm{~km}$ $\mathrm{s}^{-1}$ in the ring. For NGC 1097 and NGC 1672, the width of $[\mathrm{N} \mathrm{II}] \lambda 6584$ increases toward the nucleus, where it reaches its maximum value of $\approx 300 \mathrm{~km} \mathrm{~s}^{-1}$ for NGC 1097 and $\approx 200 \mathrm{~km} \mathrm{~s}^{-1}$ for NGC 1672. The FWHM also increases outward from the ring. In NGC 5248 the FWHM increases only slightly at the NW border of the ring, where $[\mathrm{N} \mathrm{II}] / \mathrm{H} \alpha$ ratio also increases. The gas density could be determined only for locations up to the ring (there was too much uncertainty in the $[\mathrm{S} \mathrm{II}]$ ratio farther out). For this limited distance range, it can be seen that the highest densities correspond to the loci where both $[\mathrm{N}$ II $] / \mathrm{H} \alpha$ and FWHM are highest.

As Figures 12, 13, and 14 suggest that the FWHM is correlated with the line ratio $[\mathrm{N} \mathrm{II}] / \mathrm{H} \alpha$ (and also $[\mathrm{S} \mathrm{II}] / \mathrm{H} \alpha)$, we have plotted in Figure 15 the $[\mathrm{N}$ II $] / \mathrm{H} \alpha$ ratio versus the FWHM of the [N II] $\lambda 6584$ line. In order to study the behavior of the correlation also as a function of distance 

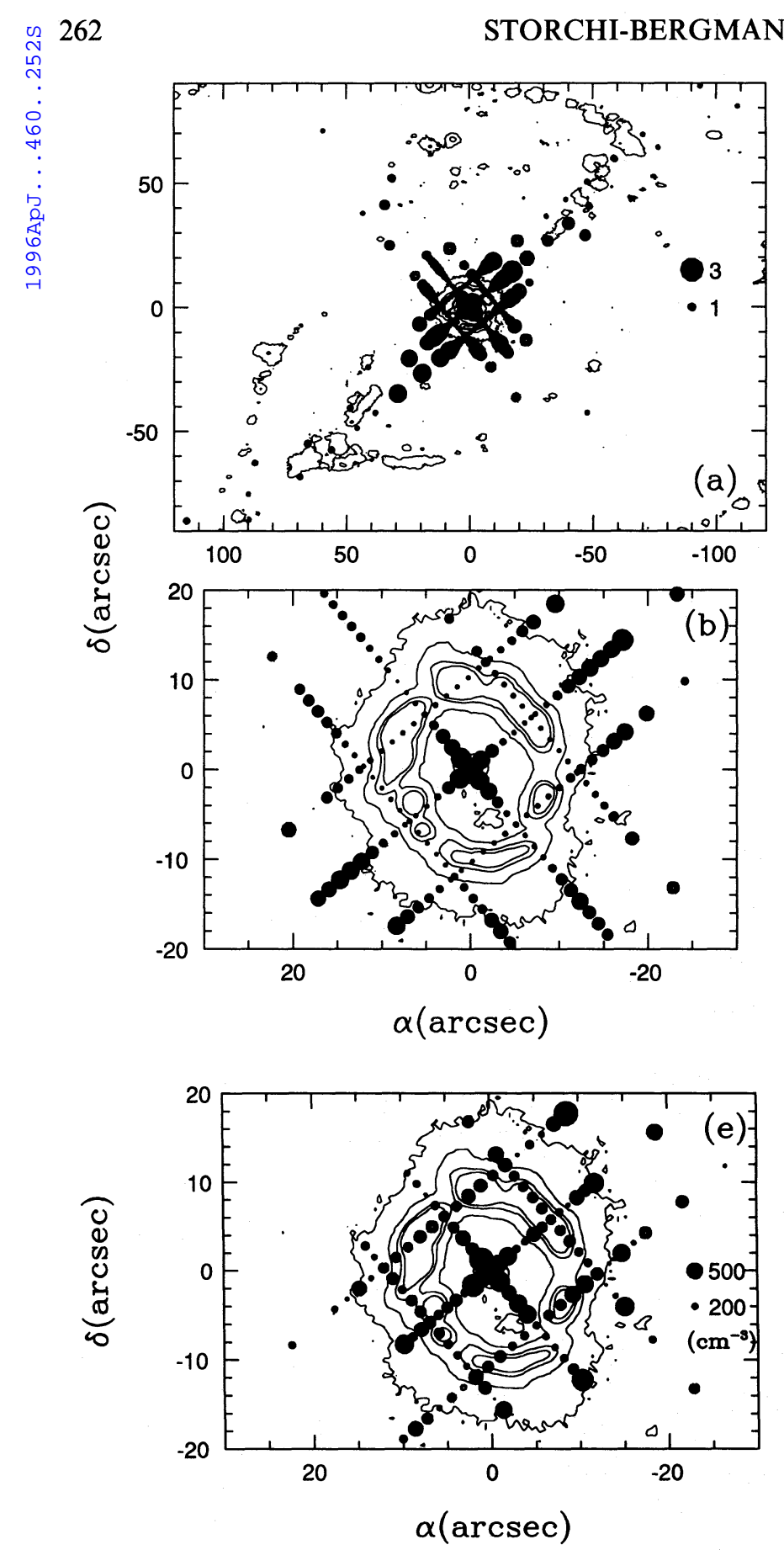

FIG. 12.-(a) The filled circles represent the emission-line ratio [N II] $\lambda \lambda 6548,6584 / \mathrm{H} \alpha$ superimposed on the continuum subtracted $\mathrm{H} \alpha$ image of NGC 1097, where the radii of the circles are proportional to the line ratios. (b) Same as (a), but enlarged to show details of the nuclear region. (c) Same as $(b)$ for the ratio [S II] $\lambda \lambda 6717,6731 / \mathrm{H} \alpha .(d)$ The circle radii are proportional to the FWHM of the [N II] $\lambda 6584$ emission line. (e) The circle radii are proportional to the gas density.

from the nucleus, we have represented different locations by different symbols: filled circles represent locations between the nucleus and the outer edge of the ring (including the nucleus), filled triangles represent locations farther than $25^{\prime \prime}$ from the nucleus, and open circles represent locations between the ring and $25^{\prime \prime}$ from the nucleus. Good correlations are observed for the inner points, with Spearman rank correlation coefficients " $R$ " of $0.8,0.7$, and 0.6 , respectively, for NGC 1097, 1672, and 5248. For locations beyond the rings, the correlation is lower. In the case of NGC 1097, there is some correlation $(R \sim 0.6)$ only when we consider
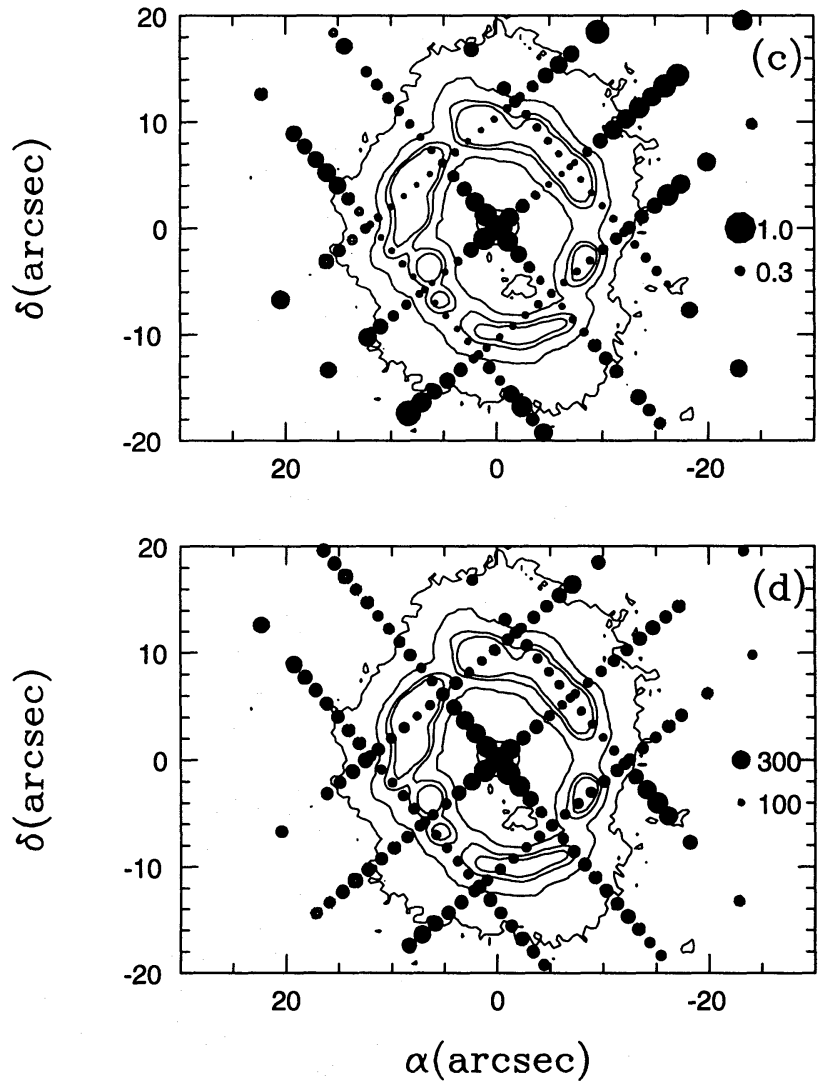

only the points farther than $25^{\prime \prime}$ from the nucleus, while for NGC 1672 a similar correlation $(R \sim 0.6)$ is observed when we consider all points beyond the ring. For NGC 5248 no correlation is found for locations beyond the ring.

The correlation between $[\mathrm{N} \mathrm{II}] / \mathrm{H} \alpha$ and the FWHM of [N II] $\lambda 6584$ for the circumnuclear gas suggests the line excitation and broadening mechanisms may be related. A similar correlation has been found in NGC 1386 (Weaver, Wilson, \& Baldwin 1991). It may suggest the presence of shock waves, which can, in principle, both ionize the gas and broaden the emission lines (Sutherland, Bicknell, \& Dopita 1993).

We have also found high $[\mathrm{N} \mathrm{II]} / \mathrm{H} \alpha$ (up to almost 3 in NGC 1097) and $[\mathrm{S} \mathrm{II}] / \mathrm{H} \alpha$ ratios well outside the nuclear rings in all three galaxies. It is unclear whether this gas is confined to the galaxy disk. Extended (nonnuclear) gas with high $[\mathrm{N} \mathrm{II}] / \mathrm{H} \alpha$ ratios has been observed in a number of galaxies. In the nearby edge-on spiral galaxy NGC 891, Keppel et al. (1991) found ionized gas up to $1.4 \mathrm{kpc}$ from the plane, with $[\mathrm{N} \mathrm{III}] / \mathrm{H} \alpha$ reaching 1.3 at the largest distances. Keppel et al. (1991) point out that this phenomenon is also observed in the Milky Way and remark that, in both cases, the ionization mechanism remains unknown. A qualitatively similar behavior is also found for the "ionized interstellar froth" observed by Hunter \& Gallagher (1990) in large-scale filaments lying outside classical $\mathrm{H}$ II regions in Magellanic irregular galaxies. [N II $],[\mathrm{S} \mathrm{II}]$, and [O II] are enhanced in the "froth" relative to $\mathrm{H} \alpha$, and exhibit larger velocity widths than those found in the $\mathrm{H}$ II regions in the same galaxies. A combination of photoionization by the OB associations (sometimes almost $1 \mathrm{kpc}$ away) and shocks is suggested as the possible ionizing mechanism.

Sokolowski, Bland-Hawthorn, \& Cecil (1991) have studied the diffuse ionized medium pervading the inner 

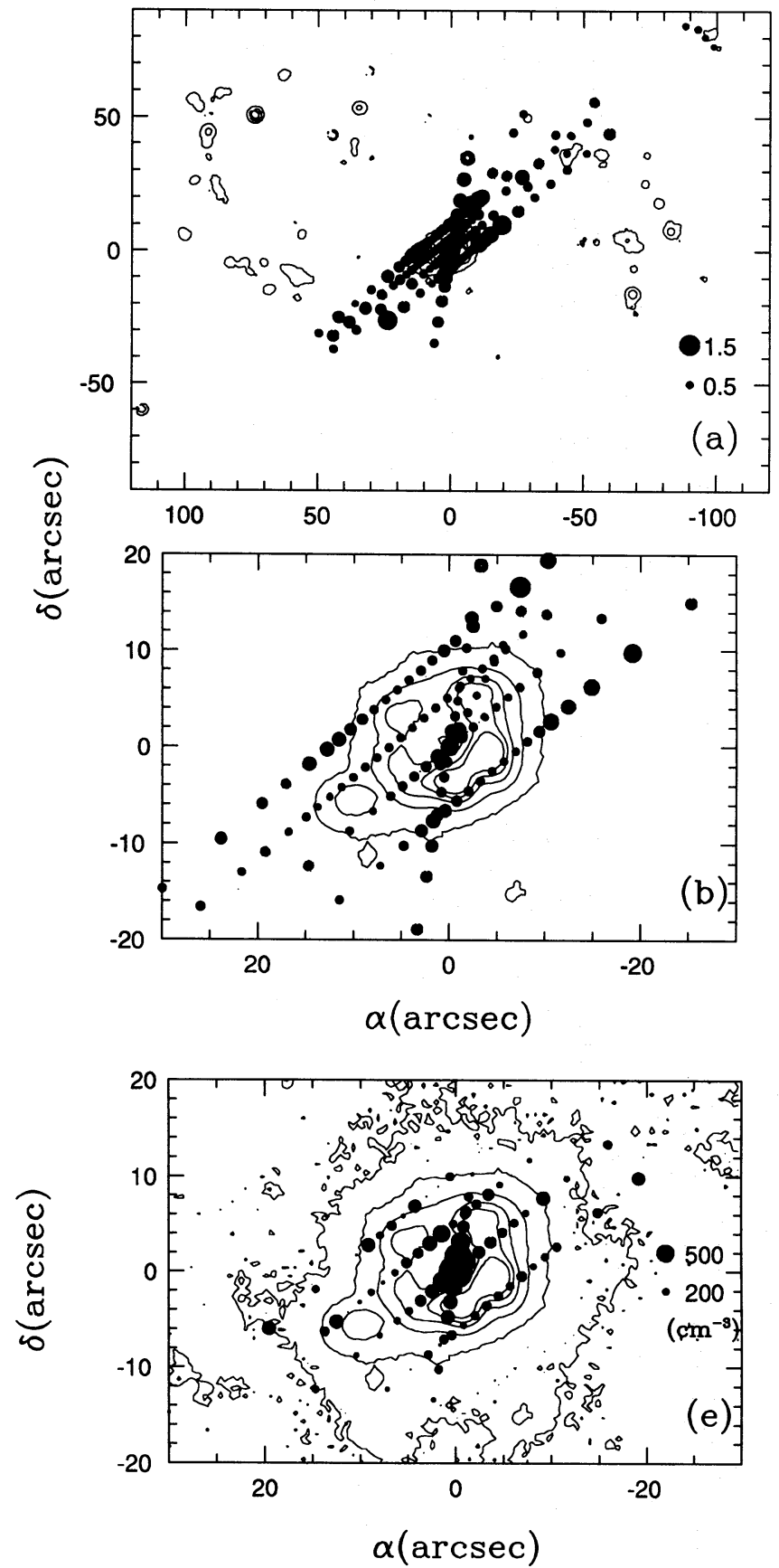

FIG. 13.-Distributions of [N II] $\lambda \lambda 6548,84 / \mathrm{H} \alpha,[\mathrm{S} \mathrm{II}] \lambda \lambda 6717,31 / \mathrm{H} \alpha$, FWHM of the line $[\mathrm{N} \mathrm{II}] \lambda 6584$ and gas density for NGC 1672 , in the same format as Fig. 12.

$10 \mathrm{kpc}$ of the disk in NGC 1068 . Here, $[\mathrm{N} \mathrm{II}] \lambda 6584 / \mathrm{H} \alpha$ ranges from 0.6 to 1.3 over most of the disk, with even larger ratios $(1.3-2.5)$ near the nucleus and radio jet axis. These authors could reproduce the high ionization, but not the low ionization (e.g., $[\mathrm{N} \mathrm{III} / \mathrm{H} \alpha$ and $[\mathrm{S} \mathrm{II}] / \mathrm{H} \alpha$ ), line ratios near the jet axis through photoionization by direct nuclear continuum light.

Within $\approx 2.5 \mathrm{kpc}$ from the nucleus, NGC 1097 has similar $[\mathrm{N} \mathrm{II}] / \mathrm{H} \alpha$ ratios to those observed in the nuclear region of NGC 1068, while for NGC 1672 the ratios are somewhat smaller and more similar to those found in the disk of NGC 1068. In NGC 5248 the range of values is similar to that observed in the "ionized interstellar froth" mentioned above. These results indicate that the excitation level of the WIM is related to the nuclear activity. The
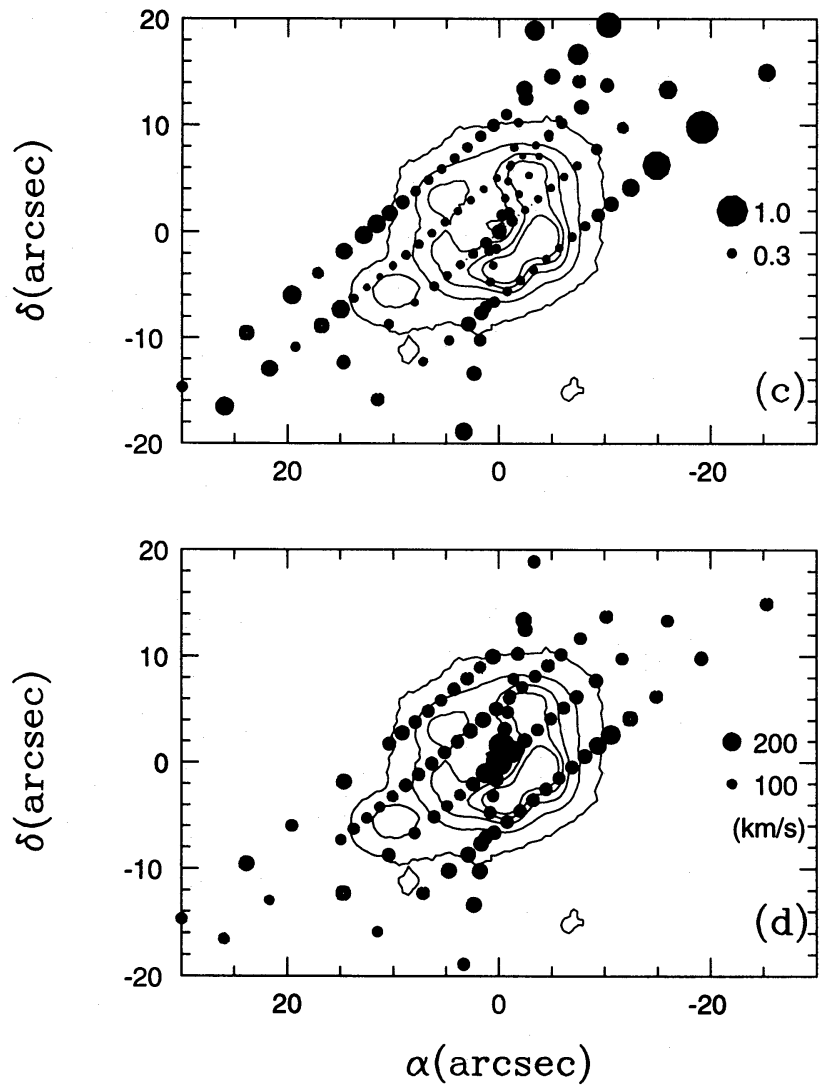

correlation observed between $[\mathrm{N} \mathrm{II}] / \mathrm{H} \alpha$ and the FWHM of [N II] suggests that at least part of the ionization is provided by shocks. The higher densities observed at the locations (inside the ring) with higher $[\mathrm{N} \mathrm{II}] / \mathrm{H} \alpha$ and FWHM also suggests that here the radiation originates in higher density gas, possibly gas compressed by the shocks. The data suggest that the shocks are present in the nuclear region of the three galaxies and farther away only for NGC 1097 and 1672.

Outside the nuclear region of NGC 5248 , the $[\mathrm{N} \mathrm{II}] / \mathrm{H} \alpha$ ratio is similar to that observed in normal galaxies. In his review, Rand (1994) points out that WIMs may be a general feature of spiral galaxies and that ionization by disk $\mathrm{O}$ stars is the most viable way of maintaining them (see also Mathis 1986).

\section{CHEMICAL ABUNDANCES}

We have measured the emission-line fluxes from the lowresolution spectra in order to calculate the chemical abundance of the gas. Values for the measured fluxes from the nucleus and a few representative locations along the slit-at the nucleus, between the nucleus, and nuclear ring, at the ring, outside the ring and at external $\mathrm{H}$ II regions - are listed in Tables 5, 6, and 7 for each galaxy. Errors in the listed line fluxes are $\leq 10 \%$ for fluxes $\geq 10^{-15} \mathrm{ergs} \mathrm{cm}^{-2} \mathrm{~s}^{-1}$, and $10 \%-30 \%$ for smaller fluxes, not including the effects of differential atmospheric refraction, which were described in $\S 3$. A few fluxes in which the error was $\geq 50 \%$ are indicated by a colon.

The line fluxes were corrected for reddening by means of the emission-line Balmer decrement $\mathbf{H} \alpha / \mathbf{H} \beta$, assuming an intrinsic decrement of 2.9. As our spectra have low $\mathrm{H} \beta$ equivalent widths at several positions, it is necessary to correct the flux of this line for underlying absorption from 

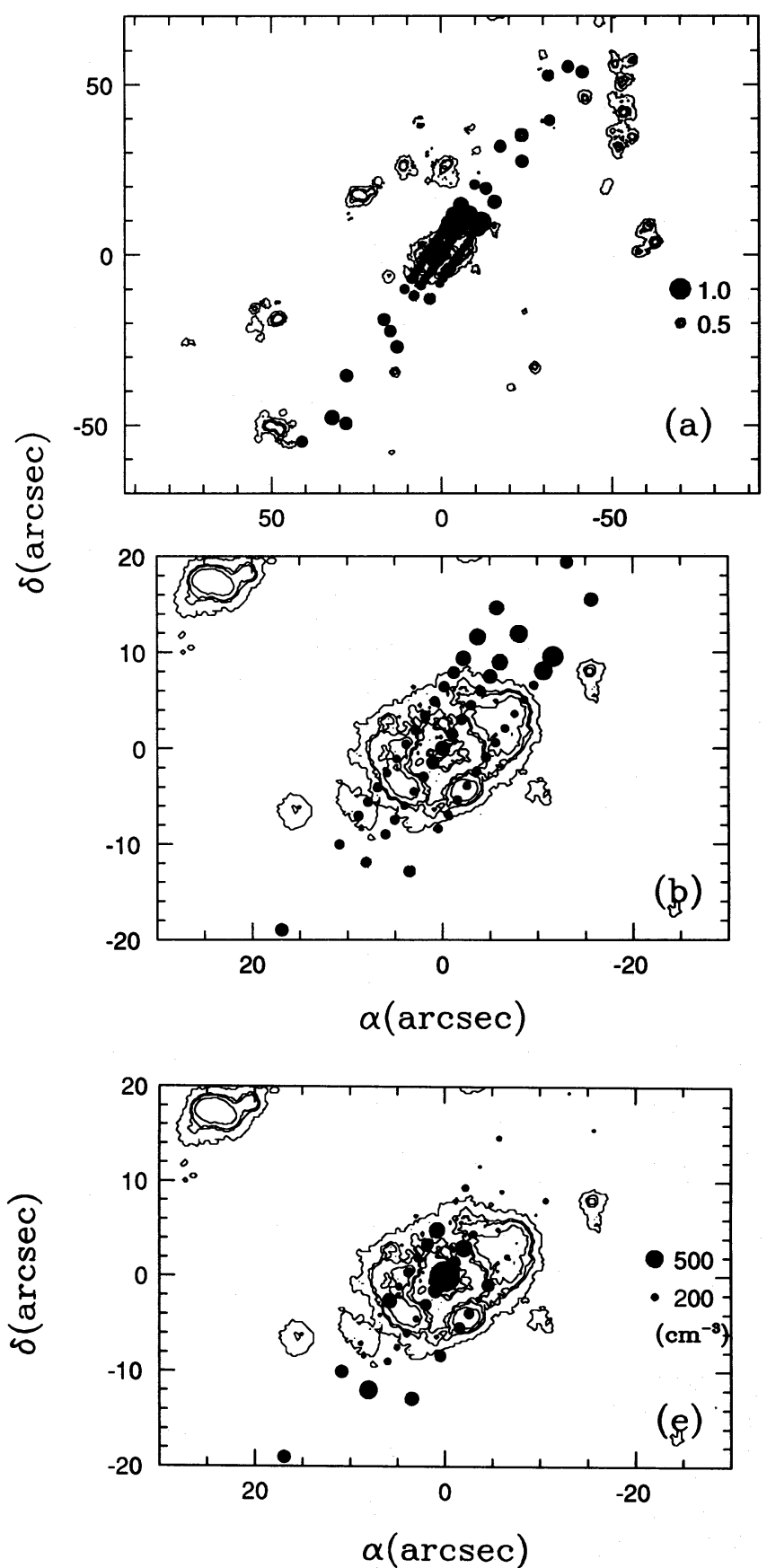

Fig. 14.-Distributions of [N II] $\lambda \lambda 6548,84 / \mathrm{H} \alpha,[\mathrm{S}$ II $] \lambda \lambda 6717,31 / \mathrm{H} \alpha$, FWHM of the line [N II] $\lambda 6584$ and gas density for NGC 5248, in the same format as Fig. 12.

the stellar population (e.g., McCall et al. 1985; StorchiBergmann, Calzetti, \& Kinney 1994, hereafter SCK). This correction was made using the following method: for each extracted spectrum, we have selected from the library of Bica (1988) the stellar population template which best matches the equivalent widths (EW) of the absorption lines which are uncontaminated by emission lines. Then, we adopted the absorption-line EWs of $\mathrm{H} \alpha, \mathrm{H} \beta$ for each bestfitting template and used these values to correct the emission-lines fluxes. The values thus obtained for the gaseous reddening are listed in column (5) of Tables 8, 9, and 10.

Emission lines were detected at the nucleus and at a number of locations along the slit. Here we use these lines to determine the gaseous chemical abundances in the ring and
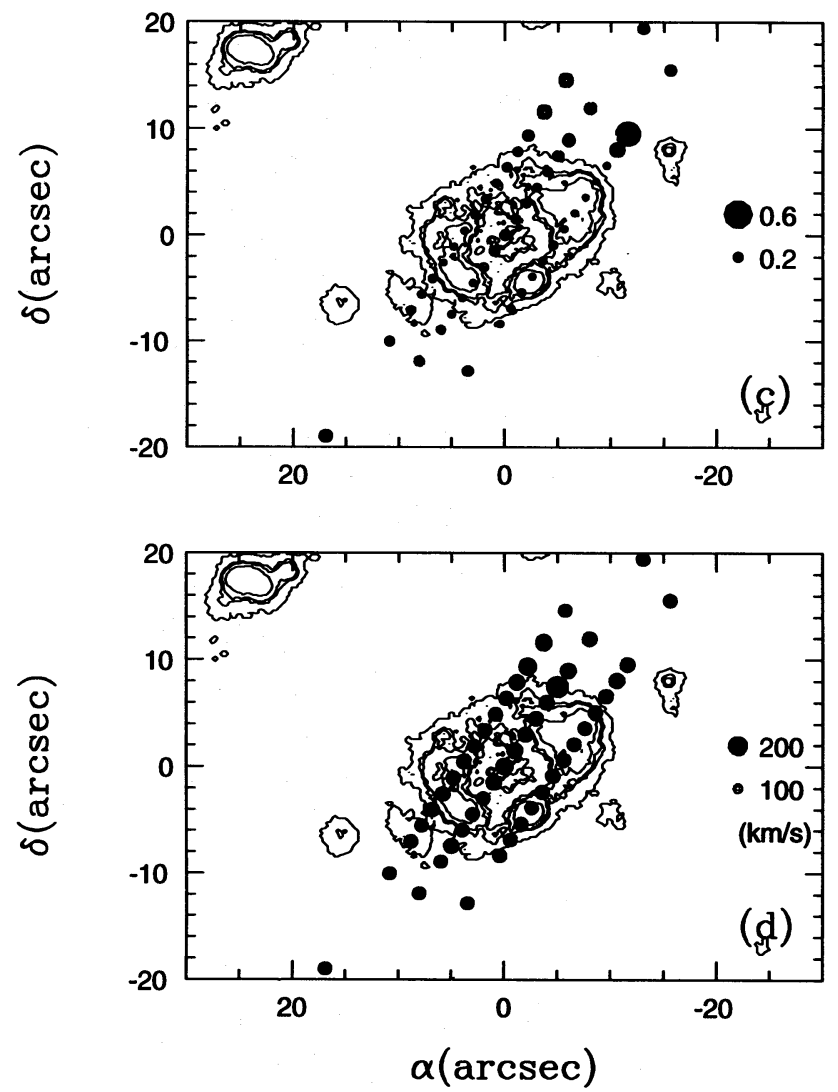

$\mathrm{H}$ II regions along the bar and/or spiral arms. In order to separate the $\mathrm{H}$ II regions from gas excited by mechanisms other than photoionization by hot stars, we have used diagnostic emission-line ratios, like $[\mathrm{O}$ III $] / \mathrm{H} \beta$ and $[\mathrm{N} \mathrm{II}] / \mathrm{H} \alpha$ (e.g., Baldwin, Phillips, \& Terlevich 1981). We emphasize the importance of such separation, as contamination by Seyfert or LINER-like emission will lead to spurious temperatures and abundances for the $\mathrm{H}$ II regions.

In the calculations of the $\mathrm{H}$ II regions abundances, a twozone model was adopted (Garnett 1992; Campbell, Terlevich, \& Melnick 1986), in which $T[$ O III] - usually obtained from the emission-line ratio [O III] $\lambda \lambda 4959,5007 / \lambda 4363$-is the temperature of the zone of high-ionization species. For the lower ionization species, such as $\mathrm{O}^{+}, \mathrm{N}^{+}$, and $\mathrm{S}^{+}$, the temperature could, in principle, be determined from the ratio [N II $] \lambda \lambda 6548,6584 /[\mathrm{N}$ II $] \lambda 5755$ (hereafter $T[\mathrm{O}$ II $]$ ). However, the temperatures are too low for us to measure [O III] $\lambda 4363$ or [N II] $\lambda 5755$ in any of our spectra, so we have used the calibration diagrams of Pagel et al. (1979), improved for lower temperatures by Vila-Costas \& Edmunds (1993), to obtain $T[\mathrm{O} \mathrm{mI}]$, which is listed in column (3) of Tables 8-10. T[O II] was obtained from the parameterization as a function of $T[\mathrm{O}$ III] given by Campbell et al. (1986). The gas density was determined from the [S II] $\lambda 6717 /[\mathrm{S} \mathrm{II}] \lambda 6731$ ratio, using the relations given by McCall (1984) and adopting T[O II] as the temperature in the $\mathrm{S}^{+}$zone. The values obtained for the density and ionic abundances are listed in Tables 8-10. The total abundances relative to solar were then calculated. Oxygen was assumed to be present in the nebulae in two ionization stages, $\mathrm{O}^{+}$ and $\mathrm{O}^{++}$, and thus the total oxygen abundance is the sum of the abundances of these two ions. Given the uncertainties in the measurements of the emission lines, the estimated uncertainties on the abundance values are $\sim 0.1$ dex.

In column (11) of Tables 8-11, we give the ratio of the nitrogen to oxygen abundance (in solar units), assuming 


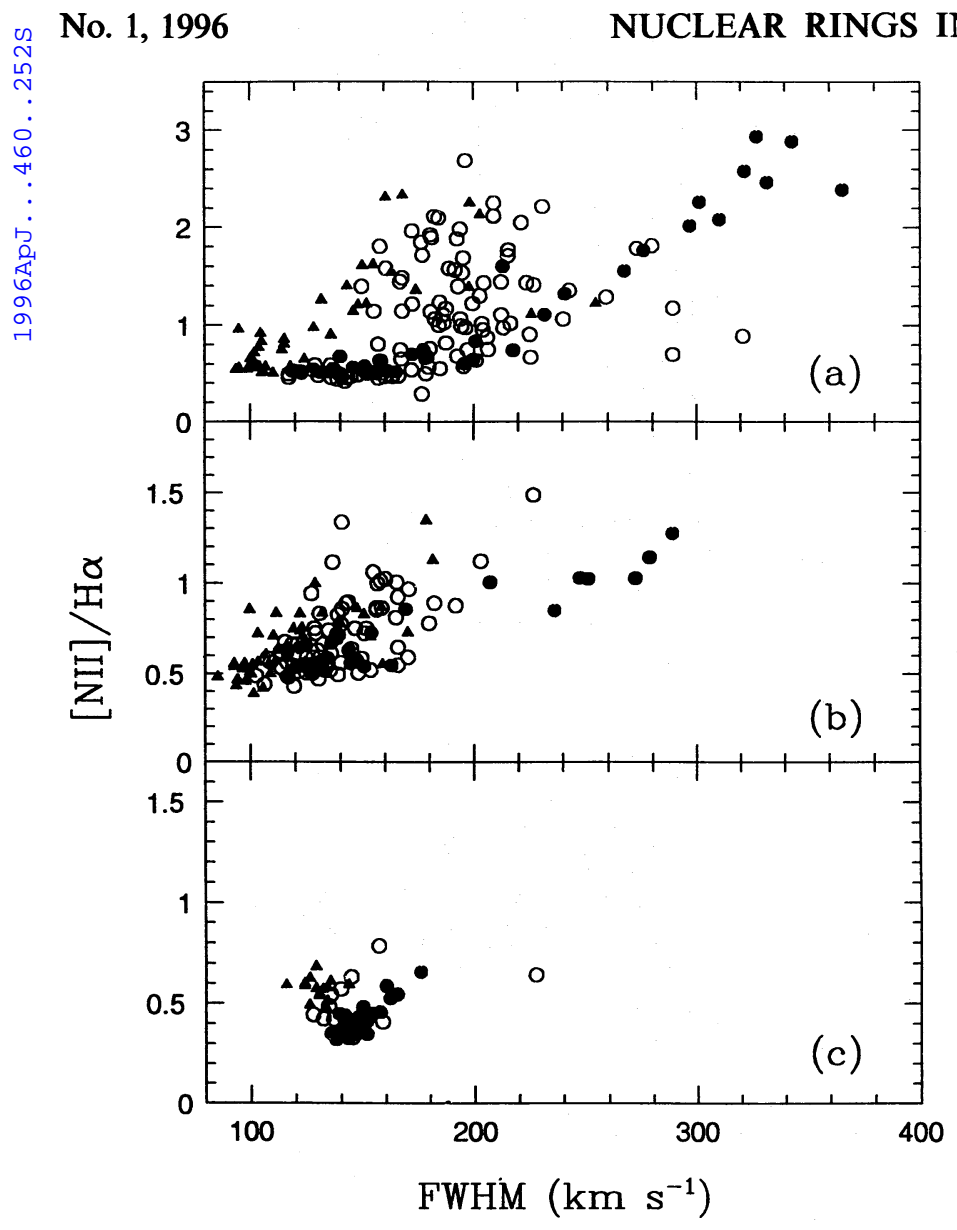

FIG. 15.-The emission-line ratio [N $\mathrm{N}] \lambda \lambda 6548,84 / \mathrm{H} \alpha$ plotted as a function of the FWHM of the [N II] $\lambda 6584$ line: (a) NGC 1097, (b) NGC 1672, (c) NGC 5248. Filled circles represent data points from locations in the ring and inside it, filled triangles represent locations farther than $25^{\prime \prime}$ from the nuclei, and open circles represent locations between the two. that $\mathrm{N} / \mathrm{O}=\mathrm{N}^{+} / \mathrm{O}^{+}$, based upon the rough coincidences between the ionization potentials of the two ions (Peimbert \& Costero 1969; Alloin et al. 1979). Recent calculations by Garnett (1990) have shown that N/O can be underestimated by this assumption due to omission of the contribution of the ion $\mathrm{N}^{++}$to the nitrogen abundance. As we do not have access to the far-infrared [ $\mathrm{N}$ III] emission lines, we will use the above approximation for $\mathrm{N} / \mathrm{O}$, keeping in mind that the true value may be $\sim 20 \%$ larger than the value listed in Tables 8-10 (Garnett 1990).

The resulting $\mathrm{O} / \mathrm{H}$ abundances are plotted as a function of distance from the nucleus in Figures $16 a, 17 a$, and $18 a$, and may be compared with the solar value of $12+$ $\log (\mathrm{O} / \mathrm{H})=8.91$. Also shown in the figure are least-squares linear fits to the measurements. The corresponding slopes and extrapolated nuclear abundances are listed in Table 11. For NGC 1097, three slopes are given: one for all locations, another for positions along the bar, and a third for positions outside the bar.

Figures $16 b, 17 b$, and $18 b$ show the dependence of the logarithm of the N/O ratio on the oxygen abundance. For comparison, two lines are also plotted on each figure, the solid one corresponding to the average sequence obtained by SCK for a sample of 44 star-forming galaxies and the dashed one to a model in which the nitrogen abundance is dominated by secondary processes (cf. Vila-Costas \& Edmunds 1993).

\subsection{Abundance Gradients}

Vila-Costas \& Edmunds (1992, hereafter VE92) have found a correlation between the metallicity gradient and the morphological type of the galaxy from observations of $\mathrm{H}$ II regions in a sample of 30 normal galaxies. They also concluded that the central abundances of spirals are correlated with their mass and that barred galaxies have shallower

TABLE 5

NGC 1097: EMISSION-LINE FLUXES ${ }^{a}$

\begin{tabular}{|c|c|c|c|c|c|c|c|c|c|c|}
\hline p.a. & $\mathbf{r}\left({ }^{\prime \prime}\right)^{b}$ & Ap. ${ }^{c}$ & $\lambda 3727$ & $\lambda 4340$ & $\lambda 4861$ & $\lambda 5007$ & $\lambda 6563$ & $\lambda 6584$ & $\lambda 6717$ & $\lambda 6731$ \\
\hline \multirow[t]{5}{*}{130} & 0 (nuc) & 9 & 16.3 & - & $1.0:$ & 14.6 & $15.6^{d}$ & 29.6 & 6.7 & 5.4 \\
\hline & 4.5 & 7.2 & 5.0 & $0.8:$ & 1.8 & 2.2 & 11.2 & 9.0 & 2.0 & 1.6 \\
\hline & 9.00 (ring) & 10.8 & 10.6 & 6.0 & 21.0 & 2.8 & 92.3 & 36.8 & 8.5 & 5.7 \\
\hline & -9.00 (ring) & 10.8 & 5.1 & 3.0 & 10.2 & 1.8 & 61.2 & 23.8 & 5.9 & 4.0 \\
\hline & -121.50 (HII) & 36 & 1.6 & 0.8 & 0.9 & $0.6:$ & 2.8 & 1.1 & 0.7 & 0.2 : \\
\hline \multirow[t]{11}{*}{139} & 0 (nuc) & 4 & 4.5 & - & 0.8 & 8.4 & $7.8^{d}$ & 22.6 & 4.4 & 4.0 \\
\hline & 8.2 (ring) & 8 & 5.1 & 4.2 & 16.3 & 1.9 & 91.1 & 35.0 & 7.3 & 6.4 \\
\hline & 49.2 (HII) & 12 & 0.8 & 0.5 & 1.5 & 0.4 & 6.9 & 3.1 & 1.5 & 1.0 \\
\hline & $55.2(\mathrm{HII})$ & 12 & 0.9 & 0.2 & 1.2 & 0.4 & 5.2 & 2.3 & 1.0 & 0.7 \\
\hline & 107.9 (HII) & 12 & 0.5 & - & 0.5 & 0.2 & 2.1 & 1.2 & 0.4 & 0.2 \\
\hline & -2 & 4 & 2.3 & - & $0.3:$ & 2.5 & 2.9 & 8.8 & 1.9 & 1.9 \\
\hline & $-9(\mathrm{HII})$ & 8 & 3.1 & 2.0 & 8.3 & 1.0 & 57.6 & 22.5 & 5.3 & 4.3 \\
\hline & -57.8 (HII) & 22 & 1.2 & 0.7 & 1.5 & 0.6 & 7.0 & 3.6 & 1.6 & 1.0 \\
\hline & -70.8 (HII) & 24 & 0.9 & 0.6 & 1.3 & 0.2 & 6.0 & 2.6 & 0.8 & 0.7 \\
\hline & -100.8 (HII) & 20 & 1.1 & - & 0.9 & 0.2 & 4.6 & 1.9 & 0.9 & 0.7 \\
\hline & $-112.8(\mathrm{HII})$ & 24 & 1.6 & - & 1.3 & 0.3 & 5.9 & 2.4 & 1.2 & 0.9 \\
\hline \multirow[t]{5}{*}{170} & 0 (nuc) & 9 & 9.1 & - & 0.8 & 11.4 & $12.0^{d}$ & 31.9 & 5.6 & 5.7 \\
\hline & 4.5 & 7.2 & 4.4 & - & 1.6 & 1.7 & 7.3 & 9.2 & 1.8 & 1.7 \\
\hline & 9.00 (ring) & 10.8 & 7.0 & 3.7 & 14.8 & 2.2 & 68.6 & 26.5 & 7.7 & 5.6 \\
\hline & -9.90 (ring) & 10.8 & 4.2 & 1.5 & 10.5 & 1.2 & 56.2 & 20.0 & 5.7 & 4.3 \\
\hline & $-83.70(\mathrm{HII})$ & 20 & 1.9 & 0.5 & 1.2 & 0.7 & 5.6 & 2.3 & 1.3 & 0.8 \\
\hline
\end{tabular}

In units of $10^{-15} \mathrm{ergs}^{-2} \mathrm{~s}^{-1}$

bositive values are to the NW of the nucleus, negative values to the SE.

c Aperture in square arcseconds of extracted spectrum.

d Narrow component. 
TABLE 6

NGC 1672: EMISSION-LiNe FluXes ${ }^{\text {a }}$

\begin{tabular}{|c|c|c|c|c|c|c|c|c|c|c|}
\hline p.a. & $r\left({ }^{\prime \prime}\right)^{b}$ & Ap..$^{c}$ & $\lambda 3727$ & $\lambda 4340$ & $\lambda 4861$ & $\lambda 5007$ & $\lambda 6563$ & $\lambda 6584$ & $\lambda 6717$ & $\lambda 6731$ \\
\hline \multirow[t]{15}{*}{94} & 0 (nuc) & 4 & 6.0 & - & 2.8 & 11.5 & 24.1 & 26.9 & 5.7 & 6.0 \\
\hline & 4 (ring) & 4 & 7.9 & 7.9 & 25.4 & 2.6 & 115.9 & 49.5 & 12.0 & 10.5 \\
\hline & 6 (HII) & 4 & 2.0 & 3.1 & 11.3 & 1.3 & 71.3 & 28.2 & 7.5 & 6.3 \\
\hline & 8 (HII) & 4 & 1.2 & 0.4 & 1.8 & 0.8 & 14.3 & 6.1 & 2.0 & 1.6 \\
\hline & 49 (HII) & 23 & 5.5 & 0.6 & 2.5 & 1.3 & 11.7 & 5.5 & 3.3 & 2.4 \\
\hline & 56 (HII) & 16 & 2.8 & 0.5 & 2.0 & 0.7 & 9.7 & 3.9 & 1.6 & 1.1 \\
\hline & 67 (HII) & 26 & 15.4 & 4.2 & 12.3 & 4.9 & 49.2 & 16.0 & 7.0 & 5.0 \\
\hline & 83 (HII) & 26 & 7.8 & 2.1 & 5.7 & 3.3 & 23.0 & 9.0 & 3.7 & 2.7 \\
\hline & -2 & 4 & 6.5 & - & 1.9 & 8.8 & 18.9 & 16.0 & 3.3 & 3.4 \\
\hline & -4 (HII) & 4 & 2.9 & 0.5 & 3.7 & 1.7 & 30.0 & 13.3 & 4.3 & 3.5 \\
\hline & -6 (HII) & 4 & 3.2 & 1.4 & 5.5 & 0.9 & 32.3 & 13.5 & 4.5 & 3.4 \\
\hline & -8 (HII) & 4 & 2.5 & 0.5 & 2.8 & 0.9 & 13.4 & 6.0 & 2.3 & 1.7 \\
\hline & -35 (HII) & 20 & 2.2 & 0.3 & 1.3 & 1.0 & 6.2 & 2.2 & 1.1 & 0.8 \\
\hline & -71 (HII) & 20 & 3.3 & 1.1 & 2.7 & 1.1 & 12.5 & 4.5 & 1.7 & 1.3 \\
\hline & -81 (HII) & 20 & 10.7 & 2.0 & 6.8 & 4.1 & 31.2 & 11.4 & 5.7 & 3.9 \\
\hline \multirow[t]{5}{*}{130} & 0 (nuc) & 7.2 & 11.6 & - & 6.2 & 12.2 & 33.7 & 30.8 & 8.4 & 7.2 \\
\hline & 3.6 (ring) & 7.2 & 9.8 & 3.8 & 16.2 & 3.8 & 69.9 & 30.2 & 9.2 & 7.7 \\
\hline & 7.2 (HII) & 7.2 & 4.7 & 2.1 & 9.0 & 1.5 & 45.2 & 17.9 & 6.3 & 4.9 \\
\hline & $58.5(\mathrm{HII})$ & 28.8 & 12.9 & 3.1 & 7.3 & 3.5 & 24.0 & 8.5 & 4.5 & 2.7 \\
\hline & 126.9 (HII) & 28.8 & 5.3 & 1.7 & 3.9 & 3.7 & 15.6 & 5.2 & 2.6 & 1.6 \\
\hline
\end{tabular}

a In units of $10^{-15} \mathrm{ergs} \mathrm{cm}^{-2} \mathrm{~s}^{-1}$.

b Positive values are to the NW of the nucleus, negative values to the SE.

c Aperture in square arcseconds of extracted spectrum.

gradients than nonbarred galaxies. Vila-Costas \& Edmunds (1993) argued that nitrogen has both a delayed primary and secondary component relative to oxygen, with the secondary component dominating at high abundance. This last result was also found in a study of the metallicity of the gas in the central regions of 44 star-forming galaxies (SCK). The method of determination of the $\mathrm{O} / \mathrm{H}$ abundance employed here is the same as used in VE92 and SCK. There is, however, a small difference in the determination of the $\mathrm{N} / \mathrm{O}$ ratio: while in the present paper and in SCK, the temperature for the region producing $\mathrm{N}^{+}$is derived from only one relation in terms of $T[\mathrm{O}$ III $]$ (Campbell, Terlevich, \& Melnick 1986), VE92 use a similar, but metallicitydependent, relation. We have, therefore, compared the results obtained from the two methods and found that the values of $\log (\mathrm{N} / \mathrm{O})$ do not differ by more than 0.05 . We thus conclude that the results presented here (Figs. 16, 17, and 18 and Table 11) can be directly compared with those from SCK and VE92.

For $T=4$, the typical central metallicities found by VE92 are $9.1 \leq 12+\log (\mathrm{O} / \mathrm{H}) \leq 9.5$. The typical gradients range from -0.03 to $-0.10 \mathrm{dex} \mathrm{kpc}^{-1}$, for nonbarred and mixed-type galaxies, while for the few barred galaxies in their sample, the gradients are $\leq-0.05$. Flatter abundance gradients in barred galaxies have also been found by other authors (e.g., Martin \& Roy 1994). The central metallicities for our three galaxies are well within the range observed by VE92. It is notable that the $\mathrm{O} / \mathrm{H}$ and $\mathrm{N} / \mathrm{O}$ abundance ratios are $0.1-0.2$ and $0.15-0.25$ dex, respectively, lower in the nucleus of NGC 5248 than in the LINER nuclei of NGC 1097 and 1672. For NGC 1097 and 5248 the metallicity gradients are near the maximum gradients observed for barred galaxies, but are also consistent with those of nonbarred and mixed-type galaxies with $T=4$. In the case of NGC 1097, Figure 16a suggests that the metallicity gradient along the bar is shallower than in other directions (consistent with mixing along the bar), although there are only three $\mathrm{H}$ II regions outside the ring and away from the bar (see Table 11). This result supports the idea that gas mixing along the bar decreases metallicity gradients. In the case of NGC 1672 (Fig. 17a), the abundances do not show a smooth gradient, but may instead be interpreted in terms of

TABLE 7

NGC 5248: EMISSION-LiNE FLUXES ${ }^{a}$

\begin{tabular}{lrrrrrrrrr}
\hline p.a. & $\mathbf{r}\left({ }^{\prime \prime}\right)^{b}$ & \multicolumn{1}{c}{ Ap. ${ }^{c}$} & $\lambda 3727$ & $\lambda 4861$ & $\lambda 5007$ & $\lambda 6563$ & $\lambda 6584$ & $\lambda 6717$ & $\lambda 6731$ \\
\hline 146 & 0 (nuc) & 10.8 & 2.2 & 2.0 & $0.6:$ & 21.6 & 9.7 & 2.3 & 2.2 \\
(through nuc.) & 5.4 (ring) & 10.8 & 1.9 & 2.3 & $0.4:$ & 21.1 & 7.5 & 2.0 & 1.5 \\
& 10.8 & 10.8 & 0.3 & 1.4 & $0.4:$ & 6.2 & 3.1 & 1.0 & 0.7 \\
& 86.4 (HII) & 36 & 7.3 & 4.9 & 1.2 & 30.6 & 12.2 & 4.4 & 3.1 \\
& -5.4 (ring) & 10.8 & 2.2 & 3.5 & $0.7:$ & 28.2 & 8.8 & 2.7 & 2.2 \\
\hline 146 & 0 (ring) & 10.8 & 2.2 & 2.6 & $0.4:$ & 26.5 & 8.7 & 2.7 & 2.1 \\
$\left(3.4^{\prime \prime} \mathrm{NE}\right)$ & 5.4 (ring) & 10.8 & 1.6 & 1.5 & $0.5:$ & 15.4 & 5.8 & 2.2 & 1.8 \\
& 10.8 (ring) & 10.8 & 1.0 & 0.8 & $0.3:$ & 6.2 & 3.1 & 0.9 & 0.6 \\
& 16.2 & 10.8 & 0.9 & 0.3 & 0.3 & 2.3 & 1.2 & 0.5 & 0.3 \\
& 65.7 (HII) & 32.4 & 1.4 & 1.0 & 0.6 & 6.5 & 3.0 & 1.0 & 0.8 \\
& 89.1 (HII) & 36 & 8.8 & 6.0 & 2.1 & 37.5 & 14.3 & 5.8 & 4.5 \\
& -5.4 (ring) & 10.8 & 1.2 & 2.5 & $0.3:$ & 20.9 & 6.2 & 2.2 & 1.8 \\
& -10.8 (HII) & 10.8 & 0.4 & 0.9 & $0.2:$ & 8.5 & 2.8 & 0.8 & 0.7 \\
& -67.5 (HII) & 36 & 1.9 & 1.5 & 0.4 & 10.6 & 4.6 & 1.6 & 1.4
\end{tabular}

a In units of $10^{-15} \mathrm{ergs} \mathrm{cm}^{-2} \mathrm{~s}^{-1}$

${ }^{b}$ Positive values are to the NW of the nucleus, negative values to the SE.

c Aperture in square arseconds of extracted spectrum. 
TABLE 8

NGC 1097: Gas Density, Temperature, Reddening, AND Abundances

\begin{tabular}{|c|c|c|c|c|c|c|c|c|c|c|}
\hline $\begin{array}{l}(1) \\
\text { p.a. }\end{array}$ & $\begin{array}{c}(2) \\
\mathrm{r}\left({ }^{\prime \prime}\right)\end{array}$ & $\begin{array}{c}(3) \\
\mathrm{T}([\mathrm{OIII}])\end{array}$ & $\begin{array}{c}(4) \\
\mathrm{N}_{e}\left(\mathrm{~cm}^{-3}\right)^{a}\end{array}$ & $\begin{array}{c}(5) \\
\mathrm{E}(\mathrm{B}-\mathrm{V})\end{array}$ & $\begin{array}{c}(6) \\
\log \left(N^{+} / H^{+}\right) \\
+12\end{array}$ & $\begin{array}{c}(7) \\
\log \left(S^{+} / H^{+}\right) \\
+12\end{array}$ & $\begin{array}{c}(8) \\
\log \left(O^{+} / H^{+}\right) \\
+12\end{array}$ & $\begin{array}{c}(9) \\
\log \left(O^{++} / H^{+}\right) \\
+12\end{array}$ & $\begin{array}{c}(10) \\
\log (O / H) \\
+12\end{array}$ & $\begin{array}{c}(11) \\
\mathrm{N} / \mathrm{O}^{b}\end{array}$ \\
\hline \multirow[t]{4}{*}{130} & 9 & 3668 & - & 0.26 & 8.35 & 6.98 & 8.91 & 8.97 & 9.24 & 2.3 \\
\hline & 101.7 & 6234 & - & 0.02 & 7.79 & 6.46 & 8.61 & 8.12 & 8.73 & 1.3 \\
\hline & -9 & 3996 & - & 0.60 & 8.24 & 6.91 & 8.91 & 8.79 & 9.15 & 1.8 \\
\hline & -121.5 & 5818 & - & 0.02 & 7.83 & 6.83 & 8.61 & 8.35 & 8.80 & 1.4 \\
\hline \multirow[t]{10}{*}{139} & 8.2 & 3595 & 218 & 0.57 & 8.36 & 7.02 & 8.92 & 9.02 & 9.28 & 2.3 \\
\hline & 49.2 & 4138 & - & 0.40 & 8.26 & 7.22 & 8.84 & 8.92 & 9.18 & 2.3 \\
\hline & 55.2 & 4286 & - & 0.30 & 8.22 & 7.15 & 8.86 & 8.78 & 9.12 & 2.0 \\
\hline & 107.9 & 4690 & - & 0.20 & 8.33 & 7.09 & 8.81 & 8.60 & 9.02 & 2.3 \\
\hline & -9 & 3778 & 158 & 0.74 & 8.31 & 7.01 & 8.95 & 8.82 & 9.19 & 1.9 \\
\hline & -57.8 & 4732 & - & 0.42 & 8.17 & 7.10 & 8.78 & 8.64 & 9.02 & 2.1 \\
\hline & -65.8 & 3800 & - & 0.38 & 8.32 & 7.12 & 8.91 & 8.90 & 9.21 & 2.2 \\
\hline & -70.8 & 4028 & 237 & 0.33 & 8.26 & 7.08 & 8.94 & 8.76 & 9.16 & 1.8 \\
\hline & -100.8 & 5621 & 191 & 0.46 & 7.96 & 6.99 & 8.77 & 8.02 & 8.84 & 1.3 \\
\hline & -112.8 & 5237 & - & 0.33 & 8.03 & 7.04 & 8.81 & 8.17 & 8.90 & 1.4 \\
\hline \multirow[t]{3}{*}{170} & 9 & 3795 & - & 0.40 & 8.29 & 7.04 & 8.91 & 8.92 & 9.22 & 2.0 \\
\hline & -9.9 & 3719 & - & 0.54 & 8.27 & 7.03 & 8.94 & 8.88 & 9.21 & 1.8 \\
\hline & -83.7 & 6170 & - & 0.37 & 7.82 & 6.85 & 8.61 & 8.17 & 8.74 & 1.4 \\
\hline
\end{tabular}

${ }^{a}$ No value is listed when the gas is the low-density limit.

${ }^{b}$ Ratio of the abundances of the two elements in units of the solar ratio.

two values of metallicity: $12+\log (\mathrm{O} / \mathrm{H}) \approx 9.2$ in the ring and $\approx 8.8 \pm 0.2$ at $3-7 \mathrm{kpc}$ from the nucleus in the bar region.

In Figures $16 b, 17 b$, and $18 b$ we plot $\log (\mathrm{N} / \mathrm{O})$ versus $12+\log (\mathrm{O} / \mathrm{H})$ together with two sequences: the average sequence obtained by SCK for starburst galaxies and the model which best fits the data of VE92 and invokes both a primary and a secondary component of nitrogen. We point out that most of the VE92 data points lie within a \pm 0.2 dex spread about the average sequence, and the SCK sequence for the starbursts represents approximately the upper limit of this spread. Most of our abundances lie between the VE92 and SCK sequences and are consistent with a mostly secondary origin for nitrogen $[\log (\mathrm{N} / \mathrm{O}) \propto \log (\mathrm{O} / \mathrm{H})]$ at the high-abundance end for NGC 1097 ad NGC 1672. The $\mathrm{N} / \mathrm{O}$ ratios are comparable to the highest values obtained by VE92 for a given $\mathrm{O} / \mathrm{H}$, but are smaller than those for the nuclear starbursts. The value of N/O is approximately constant for $12+\log (\mathrm{O} / \mathrm{H}) \leq 9.0$.

\section{ABUNDANCES IN THE ACTIVE NUCLEI}

The gaseous abundances at the nuclei can be obtained by extrapolation of the fitted curves to zero radius. Table 11 shows that the nuclear oxygen abundance $\mathrm{O} / \mathrm{H}$ ranges between 1.4 times solar (for NGC 5248) and 2.3 (for NGC 1097). The nuclear values of $\mathrm{N} / \mathrm{O}$ range between 1.7 (for NGC 5248) and 3.0 times solar (for NGC 1672). Thus the $\mathrm{N} / \mathrm{H}$ abundance is $5.3,5.6$, and 2.5 times solar for the nuclei of NGC 1097, 1672, and 5248, respectively. Because of the proximity of the rings to the nuclei, the derived nuclear abundances are within the range obtained for the rings.

Assuming that the above abundances apply to the narrow-line regions of the active nuclei, we have compared the observed nuclear-line ratios with a number of models in order to investigate the nature of the ionization source. For NGC 5248, the nuclear emission-line ratios are very similar to those from the $\mathrm{H}$ II regions in the ring and can be understood in terms of photoionization by hot stars. For NGC 1097 and NGC 1672 the nuclear ratios are very different

TABLE 9

NGC 1672: Gas Density, Temperature, Reddening, and Abundances

\begin{tabular}{|c|c|c|c|c|c|c|c|c|c|c|}
\hline $\begin{array}{l}\text { (1) } \\
\text { p.a. }\end{array}$ & $\begin{array}{l}(2) \\
r\left({ }^{\prime \prime}\right)\end{array}$ & $\begin{array}{c}(3) \\
\mathrm{T}([\mathrm{OIII}])\end{array}$ & $\begin{array}{c}(4) \\
\mathrm{N}_{e}\left(\mathrm{~cm}^{-3}\right)^{a}\end{array}$ & $\begin{array}{c}(5) \\
\mathrm{E}(\mathrm{B}-\mathrm{V})\end{array}$ & $\begin{array}{c}(6) \\
\log \left(N^{+} / H^{+}\right) \\
+12\end{array}$ & $\begin{array}{c}(7) \\
\log \left(S^{+} / H^{+}\right) \\
+12\end{array}$ & $\begin{array}{c}(8) \\
\log \left(O^{+} / H^{+}\right) \\
+12\end{array}$ & $\begin{array}{c}(9) \\
\log \left(O^{++} / H^{+}\right) \\
+12\end{array}$ & $\begin{array}{c}(10) \\
\log (O / H) \\
+12\end{array}$ & $\begin{array}{c}(11) \\
\mathrm{N} / \mathrm{O}^{b}\end{array}$ \\
\hline \multirow[t]{14}{*}{94} & 02 & 3899 & 246 & 0.16 & 8.39 & 7.15 & 8.87 & 9.05 & 9.27 & 2.8 \\
\hline & 04 & 3462 & 225 & 0.37 & 8.44 & 7.17 & 8.90 & 9.10 & 9.32 & 3.0 \\
\hline & 06 & 3351 & 176 & 0.67 & 8.45 & 7.21 & 8.82 & 9.28 & 9.41 & 3.6 \\
\hline & 08 & 4604 & 171 & 0.76 & 8.13 & 6.98 & 8.83 & 8.67 & 9.06 & 1.7 \\
\hline & 49 & 6415 & - & 0.30 & 7.82 & 6.91 & 8.63 & 7.96 & 8.71 & 1.3 \\
\hline & 56 & 5285 & - & 0.35 & 7.96 & 6.87 & 8.77 & 8.28 & 8.89 & 1.3 \\
\hline & 67 & 5063 & - & 0.21 & 7.92 & 6.86 & 8.77 & 8.46 & 8.94 & 1.2 \\
\hline & 83 & 5791 & - & 0.27 & 7.86 & 6.79 & 8.65 & 8.31 & 8.81 & 1.4 \\
\hline & -4 & 4371 & 193 & 0.61 & 8.20 & 7.04 & 8.85 & 8.78 & 9.12 & 1.9 \\
\hline & -6 & 3937 & 101 & 0.50 & 8.29 & 7.12 & 8.94 & 8.78 & 9.16 & 1.9 \\
\hline & -8 & 4414 & - & 0.33 & 8.19 & 7.07 & 8.86 & 8.69 & 9.08 & 1.8 \\
\hline & -35 & 6607 & - & 0.41 & 7.67 & 6.69 & 8.55 & 8.10 & 8.68 & 1.1 \\
\hline & -71 & 4929 & 113 & 0.27 & 7.99 & 6.88 & 8.80 & 8.49 & 8.97 & 1.3 \\
\hline & -81 & 5771 & - & 0.29 & 7.83 & 6.82 & 8.66 & 8.28 & 8.81 & 1.3 \\
\hline \multirow[t]{4}{*}{130} & 3.6 & 3952 & 186 & 0.28 & 8.29 & 7.11 & 8.89 & 8.95 & 9.22 & 2.1 \\
\hline & 7.2 & 4038 & 122 & 0.50 & 8.24 & 7.10 & 8.92 & 8.79 & 9.16 & 1.8 \\
\hline & 58.5 & 5907 & - & 0.10 & 7.80 & 6.80 & 8.66 & 8.19 & 8.79 & 1.2 \\
\hline & 126.9 & 6278 & - & 0.26 & 7.70 & 6.69 & 8.49 & 8.34 & 8.72 & 1.4 \\
\hline
\end{tabular}

a No value is listed when gas is in the low-density limit.

${ }^{b}$ Ratio of the abundances of the two elements in units of the solar ratio. 
TABLE 10

NGC 5248: Gas Density, Temperature, Reddening, and Abundances

\begin{tabular}{|c|c|c|c|c|c|c|c|c|c|c|}
\hline $\begin{array}{l}(1) \\
\text { p.a. }\end{array}$ & $\begin{array}{l}(2) \\
r\left({ }^{\prime \prime}\right)\end{array}$ & $\begin{array}{c}(3) \\
\mathrm{T}([\mathrm{OIII}])\end{array}$ & $\begin{array}{c}(4) \\
\mathrm{N}_{e}\left(\mathrm{~cm}^{-3}\right)^{a}\end{array}$ & $\begin{array}{c}(5) \\
\mathrm{E}(\mathrm{B}-\mathrm{V})\end{array}$ & $\begin{array}{c}(6) \\
\log \left(N^{+} / H^{+}\right) \\
+12\end{array}$ & $\begin{array}{c}(7) \\
\log \left(S^{+} / H^{+}\right) \\
+12\end{array}$ & $\begin{array}{l}(8) \\
\log \left(O^{+} / H^{+}\right) \\
+12\end{array}$ & $\begin{array}{c}(9) \\
\log \left(O^{++} / H^{+}\right) \\
+12\end{array}$ & $\begin{array}{c}(10) \\
\log (\mathrm{O} / \mathrm{H}) \\
+12\end{array}$ & $\begin{array}{l}(11) \\
\mathrm{N} / \mathrm{O}^{b}\end{array}$ \\
\hline $\begin{array}{c}146 \\
\text { (through nuc.) }\end{array}$ & $\begin{array}{c}5.4 \\
86.4 \\
-5.4\end{array}$ & $\begin{array}{l}4816 \\
6320 \\
4245\end{array}$ & $\begin{array}{l}108 \\
- \\
202\end{array}$ & $\begin{array}{l}0.90 \\
0.66 \\
0.77\end{array}$ & $\begin{array}{l}7.99 \\
7.78 \\
8.07\end{array}$ & $\begin{array}{l}6.72 \\
6.65 \\
6.89\end{array}$ & $\begin{array}{l}8.89 \\
8.68 \\
8.94\end{array}$ & $\begin{array}{l}8.17 \\
7.71 \\
8.59\end{array}$ & $\begin{array}{l}8.96 \\
8.73 \\
9.10\end{array}$ & $\begin{array}{l}1.1 \\
1.1 \\
1.2\end{array}$ \\
\hline $\begin{array}{c}146 \\
\left(3.4^{\prime \prime} \mathrm{NE}\right)\end{array}$ & $\begin{array}{c}0.0 \\
5.4 \\
10.8 \\
65.7 \\
89.1 \\
-5.4 \\
-10.8 \\
-67.5\end{array}$ & $\begin{array}{l}4844 \\
5083 \\
4942 \\
5901 \\
6454 \\
3780 \\
3860 \\
5532\end{array}$ & $\begin{array}{c}113 \\
204 \\
- \\
185 \\
142 \\
149 \\
340 \\
235\end{array}$ & $\begin{array}{l}0.97 \\
0.88 \\
0.61 \\
0.59 \\
0.67 \\
0.76 \\
0.81 \\
0.67\end{array}$ & $\begin{array}{l}7.95 \\
7.95 \\
8.09 \\
7.90 \\
7.74 \\
8.17 \\
8.20 \\
7.95\end{array}$ & $\begin{array}{l}6.76 \\
6.87 \\
6.86 \\
6.77 \\
6.68 \\
7.04 \\
7.02 \\
6.85\end{array}$ & $\begin{array}{l}8.90 \\
8.84 \\
8.82 \\
8.68 \\
8.66 \\
8.97 \\
8.93 \\
8.79\end{array}$ & $\begin{array}{l}8.06 \\
8.25 \\
8.40 \\
8.17 \\
7.83 \\
8.76 \\
8.96 \\
8.02\end{array}$ & $\begin{array}{l}8.96 \\
8.94 \\
8.96 \\
8.80 \\
8.72 \\
9.18 \\
9.24 \\
8.86\end{array}$ & $\begin{array}{l}1.0 \\
1.1 \\
1.6 \\
1.4 \\
1.0 \\
1.4 \\
1.6 \\
1.2\end{array}$ \\
\hline
\end{tabular}

${ }^{a}$ No value is listed when gas is in the low density limit.

${ }^{b}$ Ratio of the abundances of the two elements in units of the solar ratio.

TABLE 11

Metallicity Gradients

\begin{tabular}{lrrr}
\hline \hline GALAXY & $\begin{array}{r}\text { O/H GRADIENT } \\
(\mathrm{dex} / \mathrm{kpc})\end{array}$ & $\begin{array}{r}\text { CENTRAL } \\
12+\log (O / H)\end{array}$ & $\begin{array}{r}\text { CENTRAL } \\
\log (\mathrm{N} / \mathrm{O})\end{array}$ \\
\hline NGC1097 - all locations & -0.045 & 9.27 & -0.55 \\
NGC1097 - bar only & -0.038 & & \\
NGC1097 - bar excluded & -0.055 & 9.18 & -0.45 \\
NGC1672 & -0.062 & 9.07 & -0.70 \\
NGC5248 & -0.056 & & \\
\hline
\end{tabular}
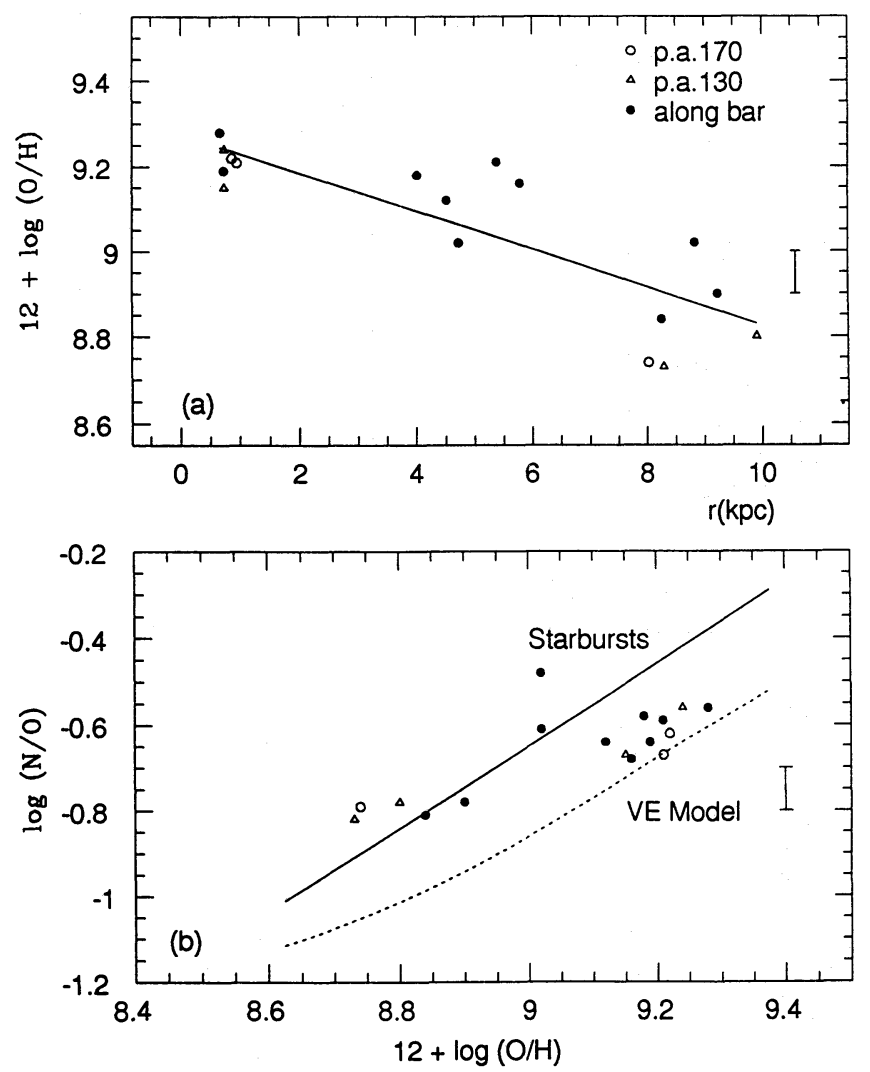

FIG. 16- (a) Oxygen abundance as a function of distance from the nucleus (in the plane of the galaxy) for NGC 1097 from data at different position angles, together with a linear least-squares fit to the points (see Table 11). (b) Relation between the nitrogen and oxygen abundances, together with the average sequence of SCK for nuclear starbursts, and the model that best fits the data of VE92 for normal spirals (considering the contribution of both primary and secondary nitrogen).
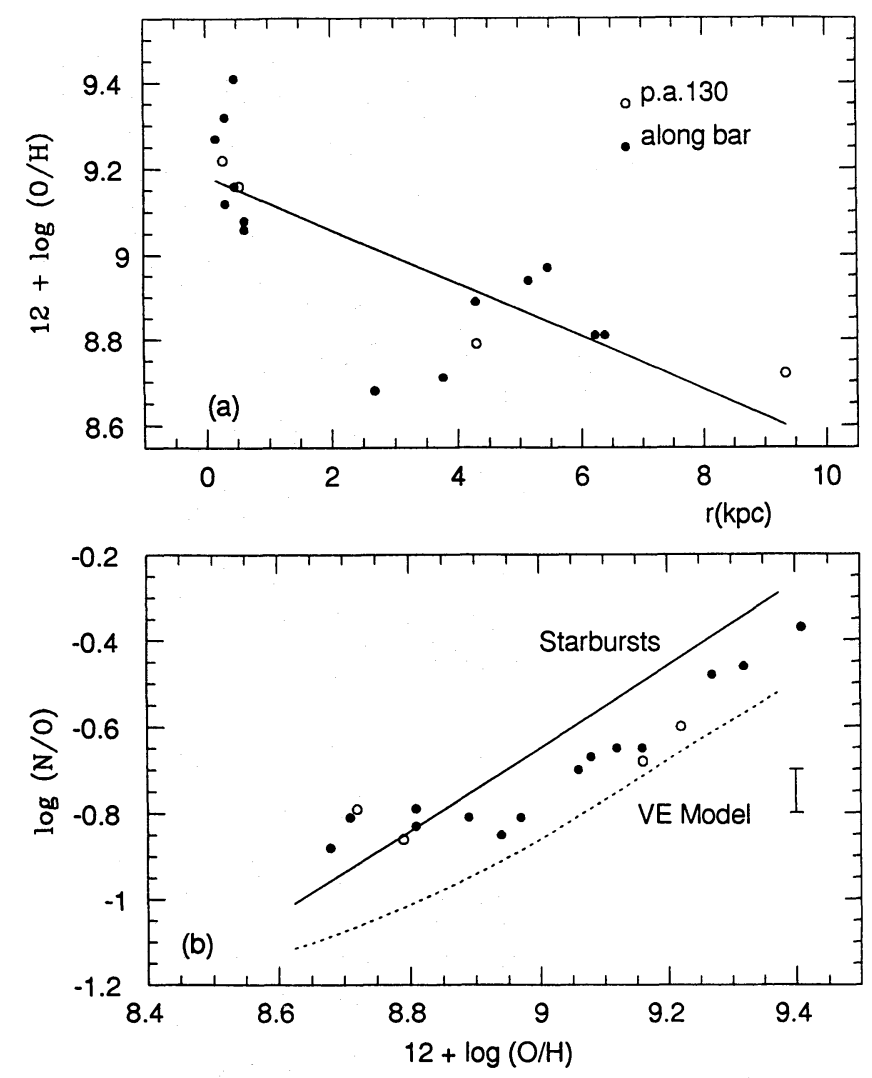

Fig. 17.-Same as Fig. 16 for NGC 1672 

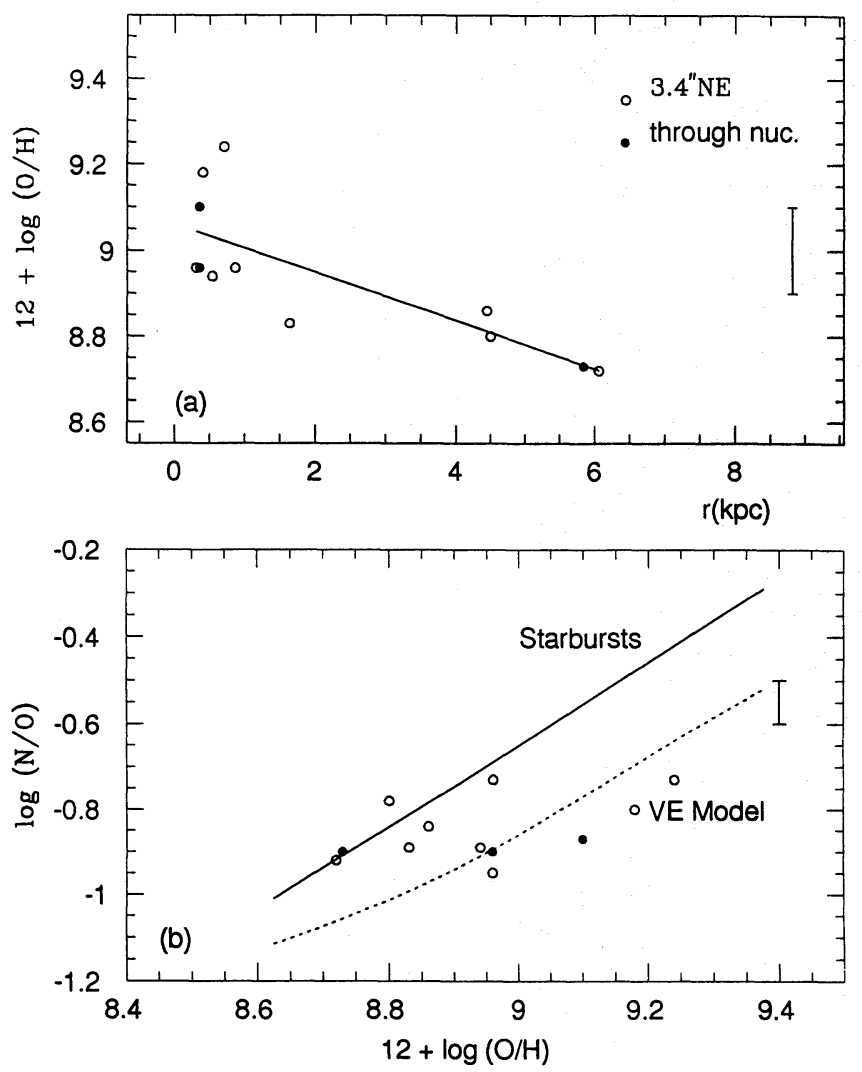

Fig. 18.-Same as Fig. 16 for NGC 5248

from those of the ring. We have considered three possibilities: (1) photoionization by an AGN continuum (Mathews \& Ferland 1987) using the program CLOUDY (Ferland 1993); (2) ionization by shocks including a radiative precursor (Dopita \& Sutherland 1995); (3) photoionization by stars with $T_{\text {eff }} \geq 45,000 \mathrm{~K}$, as suggested by Filippenko \& Terlevich (1992) and Shields (1992) to reproduce the emission-line ratios of LINERs. In all the models, the abundance of all elements except nitrogen was twice solar, while nitrogen was 5 times solar $(\approx$ the nuclear abundance derived for NGC 1097 and NGC 1672), and the gas density was taken to be $500 \mathrm{~cm}^{-3}$ (as obtained from the nuclear [S II] $26716 / \lambda 6731$ ratio).

First, we tried to model the emission-line ratios of NGC 1097. Models 1 and 2 gave too low [O $\mathrm{III}] / \mathrm{H} \beta$ and $[\mathrm{N} \mathrm{II}] / \mathrm{H} \alpha$ ratios, due to the excessive cooling in the gas because of the high abundance. We have then considered the presence of dust in model 1 (grids including the effect of dust are not available for model 2), using the option "grains" in CLOUDY, and using the depletion of heavy elements from the gas by Cowie \& Songaila (1986). We were able to get a better fit (Fig. 19) for the NGC 1097 emissionline ratios, including the nuclear $[\mathrm{O} \mathrm{I}] \lambda 6300 / \mathrm{H} \alpha$ (not shown in Fig. 19).

Nevertheless, these models do not reproduce the emission-line ratios of NGC 1672 . We retained the dust depletion, and tried model 3 with $T_{\text {eff }}=45,000 \mathrm{~K}$, with results represented by the dashed line in Figure 19. It can be seen that it reproduces the emission-line ratios of NGC 1672 quite well.

We emphasize that we have not covered all the possibilities of the models, which depend on a large number of parameters. We can only conclude that the nuclear emission-line ratios are consistent with photoionization by an AGN continuum in the case of NGC 1097 and by hot stars in the case of NGC 1672. But we point out that the temperature of the ionizing stars needed to reproduce the nuclear emission-line ratios of NGC 1672 is $\approx 10,000 \mathrm{~K}$ larger than that needed to reproduce the emission-line ratios from the rings. In addition, the correlation between $[\mathrm{N} \mathrm{II}] / \mathrm{H} \alpha$ and line width suggests that shocks may also play a role in the ionization of the gas.

\section{CONCLUSIONS}

We have studied the morphology, kinematics, excitation, and abundances of the gas in the nuclei, nuclear rings and up to several kiloparsecs from the nuclei for two galaxies with LINER nuclei-NGC 1097 and 1672-and one without-NGC 5248. The main conclusions of this paper are as follows.

1. Assuming circular motions, we have derived the kinematic parameters--position angle of the line of nodes, incli-

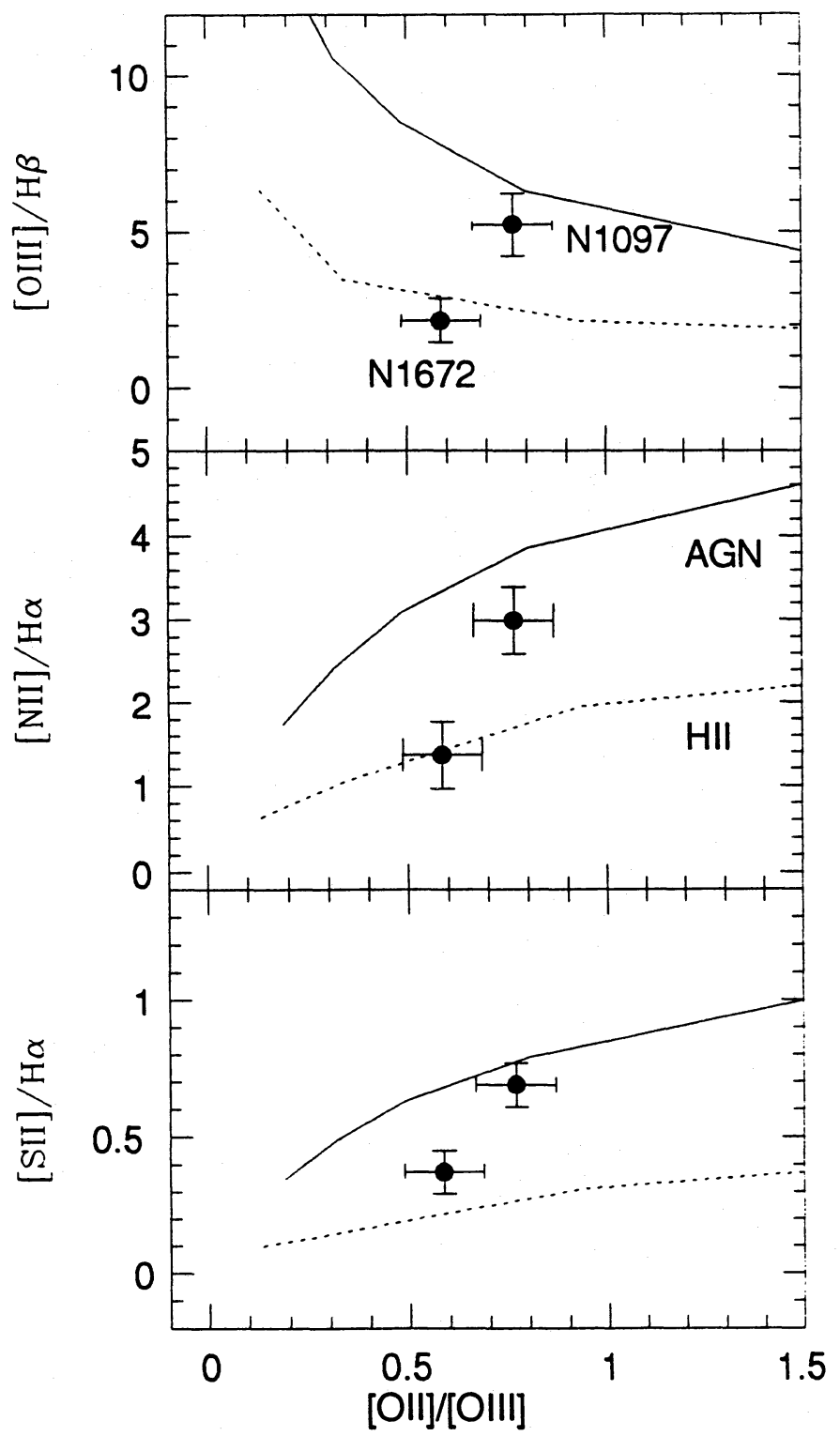

FIG. 19.-Emission-line ratios measured for the nuclei of NGC 1097 and NGC 1672 plotted together with two sequence of photoionization models (Ferland 1993), for gas with density $n=500 \mathrm{~cm}^{-3}$ and abundances 2 times solar for all elements except nitrogen, which has abundance 5 times solar. Continuous lines are for an ionizing continuum typical of an AGN, while dashed lines are for a stellar ionizing continuum with effective temperature $T=45,000 \mathrm{~K}$. In both cases, dust was included with a corresponding depletion of elements from the gas. 
nation, velocity amplitude, and radius of the turnover-for each galaxy. The turnover radii of the rotation curves for our three galaxies with nuclear rings are smaller $(\leq 0.2 \mathrm{kpc})$ than are typical $(\sim 2 \mathrm{kpc})$ of galaxies with similar morphological types and absolute magnitudes. This result suggests that the nuclear-ring galaxies have higher central mass concentrations than those without rings. The nuclear rings in NGC 1097 and 1672 are located between the two inner Lindblad resonances of the bar, if we adopt the assumption that corotation occurs at the end of the bar. The shape and orientation of the rings are in agreement with recent hydrodynamical simulations of barred galaxies (Piner et al. 1995). The fact that the ring in NGC 1097 is elongated approximately perpendicular to the bar, while in the other cases it is approximately round, suggests that the ring in NGC 1097 was formed more recently than in the other two galaxies.

2. The nuclear and circumnuclear emission-line ratios $[\mathrm{N} \mathrm{II}] / \mathrm{H} \alpha$ and $\left[\mathrm{S} \mathrm{II}_{\mathrm{II}}\right] / \mathrm{H} \alpha$ correlate well with the FWHM of the $[\mathrm{N}$ II] $\lambda 6584$ emission line, which suggests shock waves may contribute to the ionization. The emission-line ratios are, however, consistent with photoionization by a typical AGN continuum for NGC 1097, stars hotter than those ionizing the rings for NGC 1672, and stars similar to those ionizing the rings for NGC 5248.

3. The excellent sensitivity of our data has permitted the detection of emission lines from a diffuse gas-a warm ionized medium - extending to several kiloparsecs from the nuclei. The $[\mathrm{N} \mathrm{II}] / \mathrm{H} \alpha$ and $[\mathrm{S} \mathrm{II}] / \mathrm{H} \alpha$ ratios in the WIM also correlate with the FWHM of [N II] $\lambda 6584$ for the two galaxies with LINER nuclei which suggests that shocks provide at least part of the energy to ionize this diffuse gas.

4. We have calculated the gaseous abundances for the $\mathrm{H}$ II regions at the ring and beyond. The highest $\mathrm{O} / \mathrm{H}(2$ times solar) and N/O (2-3 times the solar ratio) abundances were obtained at the rings of NGC 1097 and 1672. Metal- licity gradients to $6-10 \mathrm{kpc}$ from the nucleus were also obtained and are similar to those of "normal" galaxies with the same morphological type and absolute magnitude.

5. The metallicity gradients can be extrapolated to obtain the nuclear abundances. The nuclear abundances are $(\mathrm{O} / \mathrm{H}) \approx 2.3$ and 1.9 , and $(\mathrm{N} / \mathrm{O}) \approx 2.3$ and 3.0 (in solar units) for NGC 1097 and 1672, respectively. This extrapolated abundance is within the range of metallicities of the rings (due to their proximity to the nuclei). For NGC 5248 we find central $\mathrm{O} / \mathrm{H} \approx 0.1-0.2 \mathrm{dex}$, and $\mathrm{N} / \mathrm{O} \approx 0.15-0.25 \mathrm{dex}$ lower than in the LINER nuclei. We have thus confirmed for the LINER nuclei of NGC 1097 and NGC 1672 the high abundances previously obtained for LINERs from analysis of the narrow-line regions (Storchi-Bergmann \& Pastoriza 1990; Storchi-Bergmann 1991). However, results from other works (e.g., Vila-Costas \& Edmunds 1993; SCK) suggest that comparable high abundances are found in the inner regions of nonactive galaxies of similar absolute magnitudes and morphological type, as well as in high-metallicity starbursts. There is thus no evidence, from this work, that the chemical abundances of the nuclear and circumnuclear gas have been influenced by the LINER activity, or vice versa.

We are indebted to R. Schommer for obtaining the $\mathrm{H} \alpha$ image of NGC 5248 for us and to J. Maza for obtaining one of the long-slit spectra of NGC 1097. We also acknowledge fruitful discussions with D. Calzetti, M. Dopita, L. Sparke, J. M. Stone, and P. Teuben and thank G. Ferland for making his code CLOUDY available. The referee, Linda Tacconi, made useful suggestions which helped to improve the paper. T. S. B. acknowledges the Brazilian institutions CNPq, CAPES, and FAPERGS for partial support. A. S. W. thanks NASA grants for support under grants NAGW3268 and NAGW-793. A. S. W. is also grateful to the Bergmann family and IF-UFRGS for warm hospitality.

\section{REFERENCES}

Alloin, D., Collin-Souffrin, S., Joly, M., \& Vigroux, L. 1979, A\&A, 78, 200

Arp, H. C. 1976, ApJ, 207, L147

Baldwin, J. A., Phillips, M. M., \& Terlevich, R. 1981, PASP, 93, 5

Baumgart, C. W., \& Peterson, C. J. 1986, PASP, 98, 56

Bertola, F., Bettoni, D., Danziger, J., Sadler, E., Sparke, L., \& de Zeeuw, T. 1991, ApJ, 373, 369

Bica, E. 1988, A\&A, 195, 76

Blackman, C. P. 1981, MNRAS, 195, 451

Burbidge, E. M., Burbidge, G. R., \& Pendergast, K. H. 1962, ApJ, 136, 128

Buta, R. 1987, ApJS, 64, 1

Buta, R. \& Crocker, D. A. 1993, AJ, 105, 1344

Campbell, A., Terlevich, R. J., \& Melnick, J. 1986, MNRAS, 223, 811

Corradi, R. L. M., \& Capaccioli, M. 1991, A\&AS, 90, 121

Cowie, L. L., \& Songaila, A. 1986, ARA\&A, 24, 499

de Vaucouleurs, G., de Vaucouleurs, A., \& Corwin, H. G., Jr. 1976, The

Second Reference Catalogue of Bright Galaxies (Austin: Univ. of Texas Press) (RC2)

Dopita, M. A., \& Evans, I. N. 1986, ApJ, 307, 431

Dopita, M. A., \& Sutherland, R. S. 1995, ApJ, in press

Ferland, G. J. 1993, Hazy, a Brief Introduction to CLOUDY, University of

Kentucky Physics Dept. internal report

Filippenko, A. V., \& Terlevich, R. 1992, ApJ, 397, L79

Forbes, D. A., Ward, M. J., Depoy, D. L., Boisson, C., \& Smith, M. 1992, MNRAS, 254, 509

Garcia-Gomez, D., \& Athanassoula, E. 1991, A\&AS, 89, 159

Garnett, D. R. 1990 , ApJ, 363, 142 1992, AJ, 103, 1330

Genzel, R., Weitzel, L., Tacconi-Garman, L. E., Blietz, M., Cameron, M.

Krabbe, A., Lutz, D., \& Sternberg, A. 1995, ApJ, 444, 129

Hummel, E., van der Hulst, J. M., \& Keel, W. C. 1987, A\&A, 172, 32

Hunter, D. A., \& Gallagher, J. S., III. 1990, ApJ, 362, 480

Keppel, J. W., Dettmar, R.-J., Gallagher, J. S., III, \& Roberts, M. S. 1991 , ApJ, 374, 507

Kormendy, J., \& Norman, C. A. 1979, ApJ, 233, 539

Lorre, J. J. 1978, ApJ, 222, L99

Martin, P., \& Roy, J.-R. 1994, ApJ, 424, 599

Mathews, W. G., \& Ferland, G. J. 1987, ApJ, 323, 456

Mathis, J. S. 1986, ApJ, 301, 423

McCall, M. L. 1984, MNRÁS, 208, 253

McCall, M. L., Rybski, P. M., \& Shields, G. A. 1985, ApJS, 57, 1

Ondrechen, M. P., van der Hulst, J. M., \& Hummel, E. 1989, ApJ, 342, 39

Pagel, B. E. J., Edmunds, M. G., Blackwell, D. E., Chen, M. S., \& Smith, G. 1979, MNRAS, 189, 95

Peimbert, M., \& Costero, R. 1969, Bol. Obs. Tonantzintla Tacubaya, 5, 3

Phillips, M. M., Pagel, B. E. J., Edmunds, M. G., \& Diaz, A. 1984, MNRAS, 210, 701

Piner, B. G., Stone, J. M., \& Teuben, P. 1995, ApJ, 449, 508

Pogge, R. 1989, ApJS, 71, 433

Rand, R. J., 1994, in Gas Disks in Galaxies, ed. J. M. van der Hulst, in press

Rubin, V. C., Burstein, D., Kent Ford, W., Jr., \& Thonnard, N. 1985, ApJ, 289,81

Sandage, A., \& Tammann, G. A. 1981, A Revised Shapley-Ames Catalog of Bright Galaxies, Carnegie Institution of Washington

Schwarz, M. P. 1981, ApJ, 247, 77

Sellwood, J. A., \& Sparke, L. S. 1988, MNRAS, 231, 25p

Sérsic, J. L., \& Pastoriza, M. G. 1965, PASP, 77, 287

Shields, J. C. 1992 ApJ, 399, L 30

Simkin, S. M., Su, H. J., \& Schwarz, M. P. 1980, ApJ, 237, 404

Sokolowski, J., Bland-Hawthorn, J., \& Cecil, G. 1991, ApJ, 375, 583

Sparke, L. S., \& Sellwood, J. A. 1987, MNRAS, 225, 653

Storchi-Bergmann, T. 1991, MNRAS, 249, 404

Storchi-Bergmann, T., Baldwin, J. A., \& Wilson, A. S. 1993, ApJ, 410, L11

Storchi-Bergmann, T., Calzetti, D., \& Kinney, A. 1994, ApJ, 429, 572 (SCK)

Storchi-Bergmann, T., \& Pastoriza, M. 1989, ApJ, 347, 195

. 1990, PASP, 102, 1359

Sutherland, R. S., Bicknell, G. V., \& Dopita, M. A. 1993, ApJ, 414, 510

Telesco, C. M., \& Decher, R. 1988, ApJ, 334, 573

Terlevich, R. J. 1990, in Windows on Galaxies, ed. G. Fabbiano, J. Gallagher, \& A. Renzini (Dordrecht: Kluwer)

Véron-Cetty, M. P., \& Véron, P. 1986, A\&AS, 66, 335

Vila-Costas, M. B., \& Edmunds, M. G. 1992, MNRAS, 259, 121 (VE92)

. 1993, MNRAS, 265, 199

Weaver, K. A., Wilson, A. S., \& Baldwin, J. A. 1991, ApJ, 366, 50

Wilson, A. S., Baldwin, J. A., Sun, S.-D., \& Wright, A. E. 1986, ApJ, 310, 121

Wilson, A. S., Helfer, T. T., Haniff, C. A., \& Ward, M. J. 1991, ApJ, 381, 79

Wolstencroft, R. D., \& Zealey, W. J. 1975, MNRAS, 173, 51P 